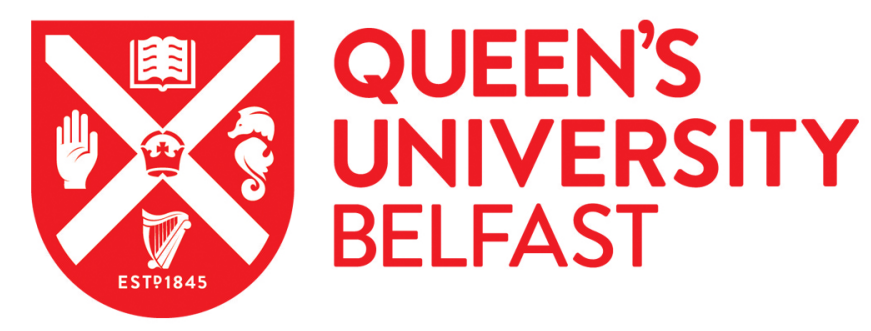

\title{
Complex organic molecules along the accretion flow in isolated and externally irradiated protoplanetary disks
}

Walsh, C., Herbst, E., Nomura, H., Millar, T. J., \& Widicus Weaver, S. (2014). Complex organic molecules along the accretion flow in isolated and externally irradiated protoplanetary disks. Faraday Discussions, 168, 389-421. https://doi.org/10.1039/C3FD00135K

Published in:

Faraday Discussions

Document Version:

Peer reviewed version

Queen's University Belfast - Research Portal:

Link to publication record in Queen's University Belfast Research Portal

Publisher rights

(C) 2014 The Royal Society of Chemistry

Final published article available at http://pubs.rsc.org/en/Content/ArticleLanding/2014/FD/C3FD00135K\#!divAbstract

\section{General rights}

Copyright for the publications made accessible via the Queen's University Belfast Research Portal is retained by the author(s) and / or other copyright owners and it is a condition of accessing these publications that users recognise and abide by the legal requirements associated with these rights.

Take down policy

The Research Portal is Queen's institutional repository that provides access to Queen's research output. Every effort has been made to ensure that content in the Research Portal does not infringe any person's rights, or applicable UK laws. If you discover content in the Research Portal that you believe breaches copyright or violates any law, please contact openaccess@qub.ac.uk. 


\title{
Complex organic molecules along the accre- tion flow in isolated and externally irradi- ated protoplanetary disks
}

\author{
Catherine Walsh, ${ }^{* a}$ Eric Herbst,${ }^{b}$ Hideko Nomura, ${ }^{c}$ T. J. Millar, ${ }^{d}$ \\ and Susanna Widicus Weaver ${ }^{e}$
}

Received Xth $X X X X X X X X X X 20 X X$, Accepted Xth $X X X X X X X X X 20 X X$

First published on the web Xth $X X X X X X X X X X 200 X$

DOI: $10.1039 / \mathbf{c 0 0 0 0 0 0 x}$

The birth environment of the Sun will have influenced the physical and chemical structure of the pre-solar nebula, including the attainable chemical complexity reached in the disk, important for prebiotic chemistry. The formation and distribution of complex organic molecules (COMs) in a disk around a T Tauri star is investigated for two scenarios: (i) an isolated disk, and (ii) a disk irradiated externally by a nearby massive star. The chemistry is calculated along the accretion flow from the outer disk inwards using a comprehensive network which includes gas-phase reactions, gas-grain interactions, and thermal grain-surface chemistry. Two simulations are performed, one beginning with complex ices and one with simple ices only. For the isolated disk, COMs are transported without major chemical alteration into the inner disk where they thermally desorb into the gas reaching an abundance representative of the initial assumed ice abundance. For simple ices, COMs can efficiently form on grain surfaces under the conditions in the outer disk. Gas-phase COMs are released into the molecular layer via photodesorption. For the irradiated disk, complex ices are also transported inwards; however, they undergo thermal processing caused by the warmer conditions in the irradiated disk which tends to reduce their abundance along the accretion flow. For simple ices, grain-surface chemistry cannot efficiently synthesise COMs in the outer disk because the necessary grain-surface radicals, which tend to be particularly volatile, are not sufficiently abundant on the grain surfaces. Gas-phase COMs are formed in the inner region of the irradiated disk via gas-phase chemistry induced by the desorption of strongly bound molecules such as methanol; hence, the abundances are not representative of the initial molecular abundances injected into the outer disk. These results suggest that the composition of comets formed in isolated disks may differ from those formed in externally irradiated disks with the latter composed of more simple ices. 


\section{Introduction}

The current accepted paradigm of isolated low-mass star formation involves the gravitational collapse of a dense condensation within a molecular cloud forming a young star surrounded by a torus of dust and gas, the protoplanetary disk.11 The dust and gas contained within the disk provide the ingredients for planets and other planetary system objects such as comets. ${ }^{2}$ However, star formation rarely occurs in isolation: stars generally form in multiple systems and within or nearby stellar clusters. $\frac{113}{13}$ One of the closest and best-studied disks, TW Hydrae, is associated with a small stellar cluster. ${ }^{4}$ There is also recent evidence that the Sun may have been born within a stellar cluster which has long since dispersed. $[5$ The nearest active high-mass star-forming region, the Orion molecular cloud (at a distance of $\approx 400 \mathrm{pc}$ ), also contains many young low-mass stars which host protoplanetary disks, so-called proplyds. 6 The proplyds in Orion are not only irradiated by the central star, they are also heavily irradiated and indeed photoevaporated by the external radiation field originating from nearby massive OB stars $\left(T_{\text {eff }} \gtrsim 10,000 \mathrm{~K}\right) \cdot{ }^{7}$

Given a large proportion of low-mass stars form within extreme environments, this raises questions regarding the influence of the external conditions on the physical and chemical structure of the disk. The birth environment may also have an impact on the planet-formation process as it is believed that planetesimals form via the coagulation of dust grains in the dense midplane. ${ }^{8}$ Ice-covered dust grains can help this process: experiments have demonstrated that the presence of the ice mantle can significantly increase the sticking probability upon collision at low relative velocities. ${ }^{9-11}$ Ice mantles also help increase the molecular complexity of the gas by providing a substrate for grain-surface chemistry to build so-called complex organic molecules (henceforth referred to as COMs) which do not often have efficient gas-phase routes to formation under typical interstellar or circumstellar conditions. ${ }^{12}$ The chemistry of COMs in protoplanetary disks is of significance because these molecules may provide the 'building blocks' of larger, more complex, prebiotic molecules, for example, amino acids. $\frac{13-15}{-15}$ it possible for such species to form within protoplanetary disks and survive assimilation into planets and comets? How does the external environment of the protoplanetary disk influence the resulting abundances and distribution of COMs? Externally irradiated disks are expected to be significantly warmer than isolated disks because the heating is dominated by the absorption of stellar and interstellar far-ultra-violet (FUV; $91.2 \mathrm{~nm}<h v<210 \mathrm{~nm}$ ) photons by dust grains. 16 Does this warmer environment facilitate or impede the production and transport of COMs in the disk? What variation in chemical complexity is expected in the planet- and comet-forming zones $(\lesssim 50 \mathrm{AU})$ of externally irradiated protoplane-

${ }^{a}$ Leiden Observatory, Leiden University, P. O. Box 9513, 2300 RA Leiden, The Netherlands. Fax: +31 71527 5819; Tel: +31 71527 6287; E-mail:cwalsh@leidenuniv.nl

${ }^{b}$ Departments of Chemistry, Astronomy, and Physics, University of Virginia, Charlottesville, VA 22904, USA.

${ }^{c}$ Department of Earth and Planetary Sciences, Tokyo Institute of Technology, 2-12-1 Ookayama, Meguro-ku, Tokyo, 152-8551, Japan.

${ }^{d}$ Astrophysics Research Centre, School of Mathematics and Physics, Queen's University Belfast, University Road, Belfast, BT7 1NN, UK.

${ }^{e}$ Department of Chemistry, Emory University, Atlanta, GA 30322, US.

2 | Faraday Discuss., [year], [vol], $1-36$

This journal is $\odot$ The Royal Society of Chemistry [year] 
tary disks compared with isolated protoplanetary disks?

The formation of COMs in a static model of an isolated T Tauri disk was investigated in previous work. ${ }^{19} \mathrm{COMs}$ were found to form efficiently via grainsurface association reactions on dust grains reaching peak fractional abundances $\sim 10^{-6}-10^{-4}$ that of the $\mathrm{H}$ nuclei number density. The physical and chemical structure of a protoplanetary disk externally irradiated by a nearby massive star was also investigated. ${ }^{20}$ Here, it was found that the disk atmosphere remains predominantly molecular in nature and the surface density is sufficient to effectively shield the midplane from the intense external FUV radiation. Consequently, the midplane temperature in the outer disk is sufficiently low $(<100 \mathrm{~K})$ for nonvolatile species (e.g., $\mathrm{H}_{2} \mathrm{O}$ and $\mathrm{CH}_{3} \mathrm{OH}$ ) to exist as ice on dust grains, potentially facilitating dust-grain coagulation and the formation of icy planetesimals.

In this work, the abundance and distribution of COMs within a typical T Tauri disk are investigated for two local environments: (i) external irradiation by the interstellar radiation field only, and (ii) external irradiation by a nearby massive O-type star. The aim is to determine the influence of the external environment on the disk structure and the resulting implications on the formation, survival, and transport of COMs. In previous work, the chemistry evolved at each grid point as a function of time, independent from neighbouring grid points. ${ }^{1920}$ Here, the chemistry is calculated along streamlines which follow the accretion flow (and transport of material) from the outer disk into the planet- and comet-forming zone $(\lesssim 50 \mathrm{AU})$. The influence of the accretion flow on the chemistry and transport of molecules within protoplanetary disks has been investigated previously. 21,24 However, this is the first investigation of the grain-surface formation and transport of COMs along the accretion flow in both an isolated disk and an externally irradiated disk.

The remainder of the paper is structured as described. In Section 2 the current observational and theoretical understanding of the chemical structure of protoplanetary disks is briefly discussed. In Section 3, the protoplanetary disk model is described, including the methods used to determine the disk physical structure (Section 3.1), the chemical network used (Section 3.2), and the method used to calculate the chemical evolution along the accretion flow (Section 3.3). In Section 4 the results of the simulations are presented and in Section 5 the main conclusions are stated.

\section{Chemistry in Protoplanetary Disks}

The complex interplay of radiation and physics in protoplanetary disks leads to disks that possess a chemically layered structure where the dominant chemical species reflect the dominant chemistry. ${ }^{25}$ Photodissociation and ionisation by UV photons and X-rays create a surface layer abundant in radicals, atoms, and ions. Shielding of the UV and X-ray radiation by dust and gas allows ionmolecule reactions to build molecular complexity in the gas phase creating a warm, molecule-rich layer deeper in the atmosphere. Towards the disk midplane, the increasing density and decreasing temperature facilitate the formation (or preservation) of ice mantles on dust grains. There is also radial stratification due to the steady increase in density and temperature moving inwards along the midplane towards the central star. As the sublimation temperature of each ice 
species is surpassed, the molecule desorbs from the grain mantle and is returned to the gas phase. The radius at which this occurs, known as the snowline, depends on the molecular binding energy of each distinct ice species. For example, the $\mathrm{CO}$ snowline is expected to reside further out in the disk than the $\mathrm{H}_{2} \mathrm{O}$ snowline because $\mathrm{CO}$ ice is more volatile than $\mathrm{H}_{2} \mathrm{O}$ ice $\left(T_{\mathrm{CO}} \approx 20 \mathrm{~K}\right.$ versus $\left.T_{\mathrm{H}_{2} \mathrm{O}} \approx 100 \mathrm{~K}\right)$ which is postulated to have implications on the $\mathrm{C} / \mathrm{O}$ ratios in forming planetesimals. 26

The physical conditions in the cold, outer disk ( $>10 \mathrm{AU}$ ) appear favourable to the production of molecules at least as complex as those observed in dark, molecular clouds. ${ }^{[27}$ This region is best observed at far-IR to (sub)mm wavelengths where many molecules emit via pure rotational transitions. However, to date, small, simple molecules only have been detected and have been limited to those species which are relatively abundant and which possess simple rotational spectra, leading to observable line emission. The molecules detected in multiple disks, thus far, include $\mathrm{CO}, \mathrm{HCO}^{+}, \mathrm{CN}, \mathrm{HCN}, \mathrm{CS}, \mathrm{N}_{2} \mathrm{H}^{+}, \mathrm{SO}, \mathrm{C}_{2} \mathrm{H}$, and associated ${ }^{13} \mathrm{C}$-, ${ }^{18} \mathrm{O}$-, and deuterium-containing isotopologues. ${ }^{428}$. 35 The detection of more complex species is hindered by the apparent size of protoplanetary disks: in astronomical terms, disks are tiny objects, on the order of a few arcseconds only in diameter. Nevertheless, current interferometric observatories have al-

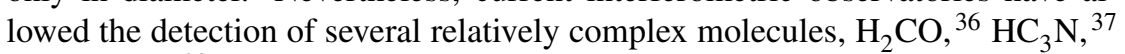
and $c-\mathrm{C}_{3} \mathrm{H}_{2} \cdot{ }^{38}$ Fortunately, the Atacama Large Millimeter/Submillimeter Array (ALMA), which is currently in its 'Early Science' phase, will have the necessary specifications to reach the sensitivities required to detect complex molecules in nearby protoplanetary disks, provided these species exist in the disk atmosphere with a sufficiently high abundance.

It is now generally accepted that most COMs form within or on ice mantles on the surfaces of dust grains. $\frac{12}{12}$ The most famous example is the simplest alcohol, methanol $\left(\mathrm{CH}_{3} \mathrm{OH}\right)$. Originally proposed some decades ago, ${ }^{39}$ it is now known that the main route to gas-phase methanol is via the desorption of methanol ice which itself forms via the sequential hydrogenation of $\mathrm{CO}$ ice,

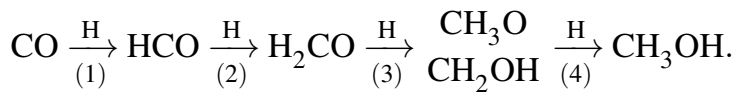

Steps (2) and (4) in the above reaction scheme are assumed to possess no reaction barrier since they involve atom addition to a radical. However, steps (1) and $(3)$ are postulated to possess large reaction barriers $(\approx 2500 \mathrm{~K})$, determined using quantum calculations. ${ }^{40}$ Laboratory studies performed at $10 \mathrm{~K}$ have shown that steps (1) and (3) have effective barriers $\approx 400 \mathrm{~K}$, suggesting the reaction sequence is assisted by the quantum tunnelling of $\mathrm{H}$ atoms through the reaction barriers. ${ }^{41 / 42}$ This mechanism efficiently forms methanol ice under dark cloud conditions reaching abundances on the order of $1 \%$ to $10 \%$ that of water ice, in line with astrophysical observations. ${ }^{43}$

Modern grain-surface chemical networks now include a plethora of atomradical and radical-radical reaction pathways to COMs and also precursor molecules

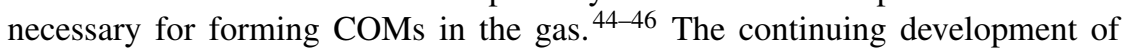
these networks has been driven by the detection of rotational line emission from multiple COMs in so-called hot cores and hot corinos. ${ }^{[12}$ These objects are thought to be the remnant molecular material leftover from the process of high-mass and

4 | Faraday Discuss., [year], [vol], 1- $\sqrt{36}$

This journal is $\odot$ The Royal Society of Chemistry [year] 
low-mass star formation, respectively. One proposed mechanism for forming COMs in these sources is that simple ices formed on grain surfaces at $10 \mathrm{~K}$ (e.g., $\mathrm{CO}, \mathrm{H}_{2} \mathrm{O}, \mathrm{NH}_{3}, \mathrm{CH}_{4}$, and $\mathrm{CH}_{3} \mathrm{OH}$ ) undergo warming to $\approx 20-30 \mathrm{~K}$ (caused by the ignition of the embedded star) where the grain-surface molecules achieve sufficient mobility for radical-radical association to form more complex species, e.g., methyl formate $\left(\mathrm{HCOOCH}_{3}\right) \cdot{ }^{44455}$ The necessary radicals are produced on the grain via dissociation by UV photons generated internally via the interaction of cosmic rays with $\mathrm{H}_{2}$ molecules. ${ }^{47}$ The importance of radiation processing for generating complex molecules in ices has been known for some time, and can be efficient at low temperatures $(10 \mathrm{~K}) \cdot \stackrel{48-50}{-5}$ Further warming to $\gtrsim 100 \mathrm{~K}$ allows those COMs formed on the grain surface to return to the gas phase thus 'seeding' the gas with complex molecules. Line emission from COMs in hot cores and hot corinos is often characterised by a gas temperature $\gtrsim 100 \mathrm{~K}$, and abundance estimates range between $\sim 10^{-10}-10^{-6}$ that of the $\mathrm{H}_{2}$ number density. 12

The formation of complex organic molecules in isolated protoplanetary disks may occur via the same mechanism given that a significant fraction of the disk material is $\gtrsim 20 \mathrm{~K}$. A fundamental difference between hot cores and protoplanetary disks is the presence of UV photons and X-rays which permeate the disk surface. In the cold, outer disk, the primary mechanism for releasing non-volatile molecules, such as, $\mathrm{H}_{2} \mathrm{O}$ and $\mathrm{CH}_{3} \mathrm{OH}$, into the gas phase is desorption triggered by the absorption of UV photons, a process termed photodesorption. ${ }^{51}$ In the disk midplane, this process is triggered by the absorption of cosmic-ray-induced UV photons. Higher in the disk atmosphere, photodesorption by stellar UV photons and X-rays and interstellar UV photons dominates. $\frac{.5253}{5}$ Photodesorption has been well-studied in the laboratory and is understood to be an indirect process. For pure $\mathrm{CO}$ ice, desorption from the ice surface is triggered by the electronic excitation of $\mathrm{CO}$ molecules in the bulk ice. ${ }^{54}$ For pure $\mathrm{H}_{2} \mathrm{O}$ ice, surface desorption is induced by photodissociation of $\mathrm{H}_{2} \mathrm{O}$ molecules deeper in the ice. $\frac{5556}{}$ In a protoplanetary disk, this generates a photodesorbed gas-phase layer of those species otherwise expected to reside on the grain at the temperatures in the outer disk, including $\mathrm{H}_{2} \mathrm{O}$ and $\mathrm{CH}_{3} \mathrm{OH}$. Indeed, photodesorption is the favoured explanation for the detection of cold $\mathrm{H}_{2} \mathrm{O}$ in the disk of TW Hya. ${ }^{\sqrt[57]{7}}$ For a protoplanetary disk externally irradiated by a nearby massive star, the disk material is significantly warmer $(>50 \mathrm{~K})$; hence, the mechanism for complex molecule formation and release into the gas phase may differ from that described above for isolated systems.

Whether COMs can form within disks also has implications on the chemical significance of comets, considered pristine material left over from the process of planet formation. ${ }^{[58}$ If COMs do not form efficiently in disks, then it is possible that comets originally consisted of simple ices which were subsequently altered (either via UV photons or thermal processes) at a more advanced phase of the evolution of the Solar System. Whether the Sun's natal protoplanetary disk was externally irradiated by nearby massive stars may also play a role in determining the attainable chemical complexity. A further consideration, not addressed in our previous work, is the composition of the material entering the protoplanetary disk. Is it possible for complex molecules to have formed at an earlier stage in the evolution of the star? If so, how are their abundances altered as they are transported through the disk? The work presented in this discussion aims to 
address these fundamental questions.

\section{Protoplanetary disk model}

The physical and chemical structure of a protoplanetary disk around a typical T Tauri star is simulated for two scenarios: (i) the isolated case, in which the disk is irradiated by FUV and X-ray photons originating from the star and interstellar medium (ISM) only, and (ii) the irradiated case, in which the disk undergoes additional irradiation by FUV photons from a nearby $(<0.1 \mathrm{pc})$ massive O-type star.

\subsection{Physical model}

The physical structure of each protoplanetary disk model is calculated using the methods outlined in previous work.$\frac{5960}{}$ Here, the important parameters only are highlighted.

The central star for both cases is a typical classical T Tauri star with mass, $M_{*}=0.5 \mathrm{M}_{\odot}$, radius, $R_{*}=0.5 \mathrm{R}_{\odot}$, and an effective temperature, $T_{*}=4000 \mathrm{~K} .61$ The disk is axisymmetric and in Keplerian rotation about the parent star. The physical structure of the disk is assumed to be steady (i.e., unchanging in time) with a constant mass accretion rate, $\dot{M}=10^{-8} \mathrm{M}_{\odot} \mathrm{yr}^{-1}$. The kinematic viscosity in the disk, $v$, is parameterised using the dimensionless $\alpha$ parameter which scales the maximum expected size of turbulent eddies by the sound speed $\left(c_{s}\right)$ and scale height $(H)$ of the disk, $v \approx \alpha c_{s} H{ }^{[62}$ For a typical T Tauri disk, $\alpha \sim 0.01$.

The dust temperature, gas temperature, and density structure of the disk are determined in a self-consistent manner by iteratively solving the necessary equations. ${ }^{63}$ Initially, the physical structure of an optically thick viscous accretion disk in hydrostatic equilibrium is adopted. ${ }^{64}$ The specific intensity everywhere in the disk, $I_{v}(r, \phi)$, is calculated by solving the axisymmetric two-dimensional radiative transfer equation for the propagation of radiation originating from the central star and external sources such as the ISM and/or a nearby massive star. The dust temperature at each position in the disk is then recalculated assuming local radiative equilibrium between the absorption and reemission of radiation by dust grains. The new gas density and temperature are determined by solving the equations of hydrostatic equilibrium in the vertical direction and detailed balance between heating and cooling of the gas, respectively. The new disk structure is adopted and the above steps performed until the convergence criterion is reached. In this way, the FUV and X-ray mean intensities are also determined at each position in the disk.

Heating sources included are radiation from the parent star and the ISM (and the nearby massive O-type star for the irradiated disk case) and the radiative flux generated by viscous dissipation in the disk midplane. ${ }^{62}$ Gas heating mechanisms included are grain photoelectric heating induced by FUV photons and $\mathrm{X}$-ray heating due to the $\mathrm{X}$-ray ionisation of $\mathrm{H}$ atoms. The gas is allowed to cool via gas-grain collisions and line transitions.

UV excess emission is commonly observed towards T Tauri stars. ${ }^{65}$ The radiation field from the central star is modelled as a combination of black body radiation at the star's effective temperature, optically thin hydrogenic bremsstrahlung

6 | Faraday Discuss., [year], [vol], $1-36$

This journal is $\odot$ The Royal Society of Chemistry [year] 
radiation at a higher temperature $\left(T_{\mathrm{br}}=25,000 \mathrm{~K}\right)$, and Lyman- $\alpha$ line emission. The total FUV luminosity from the central star is $\sim 10^{31} \mathrm{erg} \mathrm{s}^{-1}$. For the isolated disk case, external irradiation by the interstellar radiation field is also included $\left(G_{0} \approx 1.6 \times 10^{-3} \mathrm{erg} \mathrm{cm}^{-2} \mathrm{~s}^{-1}\right)$. The irradiated disk experiences additional irradiation by a nearby O-type star. The O-type star is assumed to radiate as a black body with effective temperature, $T_{\text {eff }}=45,000 \mathrm{~K}$. The FUV flux at the disk surface is set to $G_{\text {ext }}=\left(4 \times 10^{5}\right) \times G_{0}$. This corresponds to a distance between the disk and the massive star of $\lesssim 0.1 \mathrm{pc}$. Young stars are also bright in X-rays. 66 The X-ray emission from the central star for both cases is modelled as a fit to the observed XMM-Newton spectrum from the nearby classical T Tauri star, TW Hya. The total X-ray luminosity is $\sim 10^{30} \mathrm{erg} \mathrm{s}^{-1}$.

The main FUV opacity is absorption and scattering by dust grains. The dust grains are assumed to be well mixed with the gas with a gas-to-dust mass ratio $\sim 100$ and the dust-grain size distribution adopted is that which reproduces the extinction curve of dense clouds. ${ }^{[67}$ In the computation of the chemical reaction rates, for simplicity, a single dust-grain size and number density are used (see Section 3.2. The extinction of $\mathrm{X}$-rays occurs via two processes: absorption via photoionisation of all elements and Compton scattering by $\mathrm{H}$ atoms.

\subsection{Chemical model}

The physical conditions encountered in protoplanetary disks cover a wide range in density, temperature, and radiation field strength. To compute the chemistry in all regions of the disk in an appropriate manner, a network which includes all possible chemical processes must be used. The network used is the same as in previous work ${ }^{19}$ which originates from the expanded version of the Ohio State University (OSU) network. ${ }^{44}$ 46. The methods used to compute the majority of the reaction rate coefficients are the same as those outlined in previous work. $\frac{53}{5}$ The full chemical network consists of $\approx 9350$ reactions involving $\approx 800$ species. A brief description is given here.

3.2.1 Gas-phase reactions. The gas-phase network includes two-body reactions, photodissociation and photoionisation, direct cosmic-ray ionisation, and cosmic-ray-induced photodissociation and photoionisation. The core network is expanded to include direct X-ray ionisation and X-ray-induced dissociation and ionisation of elements and molecules. This is simulated by replicating the set of cosmic-ray reactions and scaling the dissociation and ionisation rates by the total X-ray ionisation rate calculated at each grid point in the disk, $\zeta_{\mathrm{xr}}(x, z)$. The photoreaction rates in the core network are normalised to the average strength of the unshielded interstellar radiation field, $G_{0}$.

Under astrophysical conditions, many molecules dissociate primarily via line absorption rather than via continuum absorption. $\frac{6970}{}$ For those species which achieve sufficiently large column densities, self shielding can dominate over shielding by dust grains. ${ }^{71}$ Self shielding occurs when foreground molecular material absorbs the photons necessary for photodissociation to occur deeper into the atmosphere. The photodissociation cross sections can also overlap with those for $\mathrm{H}_{2}$. In this work, the self- and mutual-shielding of $\mathrm{H}_{2}, \mathrm{CO}$, and $\mathrm{N}_{2}$ are taken into account. ${ }^{72-74}$

This journal is $\odot$ The Royal Society of Chemistry [year]

Faraday Discuss., [year], [vol], $1 \sqrt{36 \mid} \mid 7$ 
3.2.2 Gas-grain interactions. The gas-phase network is supplemented with gas-grain interactions to allow the condensation (freezeout) and sublimation (desorption) of molecules onto, and from, dust grains, respectively. The freezeout rate onto dust grains is dependent upon the geometrical cross section of dust grains and the velocity of the gas. It is assumed that sticking occurs upon each collision with a dust grain, i.e., a sticking coefficient equal to 1 is used for all species. $\mathrm{H}$ atoms are known to stick less efficiently to grain surfaces than other species. ${ }^{75}$ Here, the temperature dependence of the sticking coefficient for $\mathrm{H}$ atoms is taken into account. ${ }^{76177}$

The thermal desorption rate is calculated assuming the bound molecule oscillates in a harmonic potential well. ${ }^{78}$ The thermal desorption rate depends on the binding energy of the molecule to the grain surface, the temperature of the dust, and the characteristic frequency of vibration of the molecule. Volatile molecules, e.g., $\mathrm{CO}$, will desorb from the grain mantle at lower temperatures than less volatile species, e.g., $\mathrm{H}_{2} \mathrm{O}$. Thermal desorption triggered by cosmic-ray heating of dust grains is also included. ${ }^{79}$ This process is significant for volatile molecules only.

For species which are strongly bound to the grain mantle, e.g., $\mathrm{H}_{2} \mathrm{O}$, photodesorption is the primary mechanism for releasing molecules into the gas phase in the cold, outer disk. The photodesorption rate of each species depends on the flux of UV photons, the geometric cross section of a dust-grain binding site, the surface coverage of the molecule, and the molecular yield per UV photon. This process is now well studied in the laboratory and photodesorption yields are determined for $\mathrm{CO}, \mathrm{N}_{2}, \mathrm{CO}_{2}, \mathrm{H}_{2} \mathrm{O}$, and $\mathrm{CH}_{3} \mathrm{OH} \cdot \frac{50|55| 80 \mid 81]}{}$ For all other species, a yield of $10^{-3}$ molecules photon ${ }^{-1}$ is adopted, in line with those values already constrained in the laboratory. Recent experiments and simulations have also determined that photodesorption occurs from the top two monolayers of the ice mantle only, and this is accounted for in the calculation of the photodesorption rates. ${ }^{54}$ Photodesorption triggered by all sources of UV photons are included: external stellar and interstellar UV photons, and UV photons generated internally by the interaction of cosmic rays with $\mathrm{H}_{2}$ molecules.

3.2.3 Grain-surface reactions. A comprehensive grain-surface network is adopted to simulate the formation of COMs. $\stackrel{446}{46}$ The grain-surface association reactions are assumed to occur via the Langmuir-Hinshelwood mechanism only, i.e., two adsorbed species on the grain surface diffuse and react. The reaction rate coefficients are calculated using the rate equation method. ${ }^{78}$ This method is appropriate due to the relatively high densities, $\gtrsim 10^{7} \mathrm{~cm}^{-3}$, in the regions of the disk where grain-surface chemistry is important. Because of the discrete nature of dust grains, processes occurring on grain surfaces are stochastic: however, comparisons of stochastic models of grain-surface chemistry with models employing the rate equation method show that stochastic effects are most important at lower densities $\left(\lesssim 10^{5} \mathrm{~cm}^{-2}\right) .82+84$

Classical thermal diffusion is assumed for all species, except $\mathrm{H}$ and $\mathrm{H}_{2}$ which are also allowed to quantum tunnel through the diffusion barrier. This is the 'optimistic' case: recent experiments investigating the surface mobility of $\mathrm{H}$ atoms on water ice have proved inconclusive regarding the exact contribution of quantum diffusion to the total diffusion rate ${ }^{85}$ The ratio of the diffusion barrier to the binding energy of each species is assumed to be 0.3 . Again, this is an

8 | Faraday Discuss., [year], [vol], $1-36$

This journal is $\odot$ The Royal Society of Chemistry [year] 
'optimistic' value which allows the efficient diffusion and reaction of relatively volatile species at $\sim 20 \mathrm{~K}$. For each exothermic grain-surface association reaction which leads to a single product, there is a probability that the product will be returned to the gas phase. This is the process of 'reactive' or 'chemical' desorption and provides an additional desorption mechanism for non-volatile molecules. A probability of $1 \%$ is adopted in this work. $\frac{86}{6}$

3.2.4 Dust-grain model. A fixed dust grain size of $10^{-5} \mathrm{~cm}$ is adopted and the number density of dust grains is assumed to equal $\sim 10^{-12}$ that of $\mathrm{H} \mathrm{nu}$ clei. These values correspond to so-called classical grains which possess $\approx 10^{6}$ surface binding sites per grain.

3.2.5 $\mathrm{H}_{2}$ formation rate. $\mathrm{H}_{2}$ molecules also form on dust-grain surfaces and the conversion from $\mathrm{H}$ to $\mathrm{H}_{2}$ and vice versa must also be taken into account. Here, the most optimistic case is adopted: it is assumed that the rate of formation of gas-phase $\mathrm{H}_{2}$ equates to half the rate of arrival of $\mathrm{H}$ atoms onto the grain surfaces. This allows efficient formation of $\mathrm{H}_{2}$ on warm dust grains $(\gtrsim 25 \mathrm{~K})$ in line with astrophysical observations towards diffuse clouds and photon-dominated regions (PDRs) ${ }^{87 / 88}$ If the explicit grain-surface route to $\mathrm{H}_{2}$ is included, the rate of formation of gas-phase $\mathrm{H}_{2}$ drops significantly at warm temperatures ( $\gtrsim 25 \mathrm{~K}$ ) due to the volatile nature of $\mathrm{H}$ atoms. In reality, the formation rate likely lies between these two extrema. Several solutions have been suggested such as allowing both the physisorption and chemisorption of $\mathrm{H}$ atoms on dust grains, and including $\mathrm{H}_{2}$ formation via both the Eley-Rideal and Langmuir-Hinshelwood mechanisms. ${ }^{89}$ - Certainly, these solutions should be explored in future protoplanetary disk models.

Note that this work does include the temperature-dependent behaviour of the sticking coefficient of $\mathrm{H}$ atoms which is found to decrease with increasing temperature. .7677

\subsection{Accretion flow}

In previous work, the chemistry was computed at each point in the disk as a function of time, using initial abundances generated using a simple molecular cloud model.1920153192 The chemistry was set up to evolve at each grid point independent of that in neighbouring grid points, i.e., the rate of change in abundance of species $i$ at a point $(x, z)$ was given by,

$$
\frac{\mathrm{d} n_{i}(x, z)}{\mathrm{d} t}=P_{i}-D_{i} \quad \mathrm{~cm}^{-3} \mathrm{~s}^{-1}
$$

where $P_{i}$ and $D_{i}$ are the production and destruction rates of species, $i$, respectively. Here, chemical abundances are computed along the accretion flow by integrating the chemical evolution of a fluid parcel along streamlines from the outer edge of the disk inwards towards the central star. The rate of change in abundance of species $i$, is derived assuming,

$$
\frac{\partial\left(n_{i} v_{x}\right)}{\partial x}=\frac{\mathrm{d} n_{i}}{\mathrm{~d} t}=P_{i}-D_{i} \quad \mathrm{~cm}^{-3} \mathrm{~s}^{-1}
$$

This journal is @ The Royal Society of Chemistry [year]

Faraday Discuss., [year], [vol], 1-36 | 9 
where $v_{x}$ is the radial velocity along a streamline. Using the continuity equation,

$$
\frac{\partial\left(n_{\mathrm{T}} v_{x}\right)}{\partial x}=0 \quad \mathrm{~cm}^{-3} \mathrm{~s}^{-1},
$$

where $n_{\mathrm{T}}$ is the total number density, the rate of change in abundance of species $i$ with radius is given by

$$
\frac{\mathrm{d} n_{i}(x, z)}{\mathrm{d} x}=\frac{1}{v_{x}(x)} \frac{\mathrm{d} n_{i}(x, z)}{\mathrm{d} t}+\frac{n_{i}(x, z)}{n_{\mathrm{T}}(x, z)} \frac{\mathrm{d} n_{\mathrm{T}}(x, z)}{\mathrm{d} x} \quad \mathrm{~cm}^{-2} .
$$

The radial velocity of the flow is given by

$$
v_{x}(x)=-\frac{\dot{M}}{2 \pi \Sigma(x) x} \quad \mathrm{~cm} \mathrm{~s}^{-1},
$$

where $\dot{M}$ is the assumed mass accretion rate and $\Sigma(x)$ is the surface density of the disk at a radius, $x$. Streamlines, $l$, are defined as fractions of the disk scale height, i.e., $l=f H$, with $0 \leq f \leq 4$. The disk scale height is determined at the disk midplane for all streamlines,

$$
H_{0}(x)=\frac{c_{s 0}(x)}{\Omega(x)} \propto T_{0}^{1 / 2} x^{3 / 2},
$$

where $c_{s 0}(x)=\sqrt{k T_{0}(x) / m_{0}(x)}, T_{0}(x)$, and $m_{0}(x)$ are the sound speed, gas temperature, and mean molecular mass in the disk midplane, and $\Omega(x)=\sqrt{G M_{*} / x^{3}}$ is the Keplerian angular velocity at a radius, $x$. $G, M_{*}$, and $k$ represent the gravitational constant, stellar mass, and Boltzmann's constant, respectively. The disk scale height, $H$, and the geometrical height, $z$, are related via the assumption of hydrostatic equilibrium which defines the density structure, $\rho(x, z)$,

$$
\rho(x, z)=\rho_{0}(x) \exp \left(\frac{-z^{2}}{2 H(x)^{2}}\right),
$$

where $\rho_{0}(x)$ is the density in the midplane, $z=0$, at a radius, $x$. Physical conditions, such as density, temperature, and radiation field strength, are extrapolated along the flow by assuming each parameter follows a power law behaviour $\left(\propto \alpha x^{-\beta}\right)$ between grid points.

\subsection{Initial conditions}

The initial abundances injected into the outer edge of each streamline are extracted at a time of $10^{5}$ years from a simple time-dependent molecular cloud model with a fixed density of $10^{5} \mathrm{~cm}^{-3}$ and a high visual extinction of $\gg 10$ mag. The chemical model used is the same as that described in Section 3.2 A cosmicray ionisation rate of $1.3 \times 10^{-17} \mathrm{~s}^{-1}$ is assumed. The time-dependent cloud model calculation is begun assuming all species are in atomic form except hydrogen which is in molecular form. The assumed elemental abundances for $\mathrm{H}: \mathrm{He}: \mathrm{C}: \mathrm{N}: \mathrm{O}$ are 1.0:9.75(-2):1.4(-4):7.5(-5):3.2(-4). For the heavier elements, $\mathrm{H}: \mathrm{Na}: \mathrm{Mg}: \mathrm{Si}: \mathrm{S}: \mathrm{Cl}: \mathrm{Fe}$, the ratios are 1.0:2.0(-9):7.0(-9):8.0(-9):8.0(-8):4.0(-9):3.0(9).

10 | Faraday Discuss., [year], [vol], 1-36 This journal is $\odot$ The Royal Society of Chemistry [year] 
It is assumed that the material entering the outer region of the protoplanetary disk has remained shielded from the central star. Two sets of initial abundances are generated: one for the 'cold' case assuming a constant gas and dust temperature of $10 \mathrm{~K}$, and one for the 'warm' case assuming a constant temperature of $30 \mathrm{~K}$. In this way, either 'pristine' dark cloud initial abundances are used, or it is assumed a degree of thermal processing has occurred before injection into the disk. The latter value of $30 \mathrm{~K}$ was chosen based on preceding work investigating the formation and distribution of COMs in a static model of an isolated protoplanetary disk. ${ }^{19}$ In that work it was found that thermal grain-surface chemistry efficiently operates and builds COMs in the ice when the dust temperature exceeds $\approx 20-30 \mathrm{~K}$, depending on the assumed barrier to surface diffusion $(0.3-0.5$ times the desorption energy). For the lower value of the diffusion barrier, a peak in the abundances of grain-surface COMs was seen at a temperature of $\approx 30 \mathrm{~K}$; hence, this temperature is adopted for the 'warm' case. In addition, the maximum dust temperature reached at the outer edge of the isolated protoplanetary disk model is $\approx 30 \mathrm{~K}$. This higher temperature also affects the relative abundances and subsequent chemistry of volatile species, for example, CO. In the cold model, only those COMs which can form efficiently via atom-addition reactions on grain surfaces reach appreciable fractional abundances because at $10 \mathrm{~K}$ atoms alone have sufficient mobility to diffuse and react. However, at $30 \mathrm{~K}$, weakly bound molecules, such as molecular radicals, also have sufficient mobility, allowing greater complexity to build within the ice mantle via radical-radical association reactions. ${ }^{44}$ Hence, for the 'cold' case, the calculations begin with simple ices only, whereas for the 'warm' case, the calculations begin with simple and complex ices. For the warm case, cosmic-ray-induced photodesorption releases a proportion of COMs from the grain mantle so that the calculations also begin with appreciable fractional abundances of COMs in the gas phase.

The initial molecular fractional abundances (with respect to total $\mathrm{H}$ nuclei density) for COMs of interest in this work are listed in Table 1. The species highlighted here are those which have been observed in the gas phase in cold molecular clouds and/or hot cores/corinos and/or require methanol ice as a parent molecule. Also listed are the assumed binding energies for each species. $\stackrel{44}{4}$ Dimethyl ether, $\mathrm{CH}_{3} \mathrm{OCH}_{3}$, methyl formate, $\mathrm{HCOOCH}_{3}$, and glycolaldehyde, $\mathrm{HOCH}_{2} \mathrm{CHO}$, are all thought to form via the association of radicals produced either via the hydrogenation of $\mathrm{CO}$ ice, or the photodissociation of methanol ice,, $45 \sqrt{46 / 50}$

$$
\begin{aligned}
\mathrm{CH}_{3} \mathrm{OH}+h v & \longrightarrow \mathrm{CH}_{3}+\mathrm{OH}, \\
& \longrightarrow \mathrm{CH}_{3} \mathrm{O}+\mathrm{H}, \\
& \longrightarrow \mathrm{CH}_{2} \mathrm{OH}+\mathrm{H} .
\end{aligned}
$$

The relative branching ratios for these photodissociation pathways are thought to influence the resulting abundances of $\mathrm{CH}_{3} \mathrm{CHO}, \mathrm{CH}_{3} \mathrm{OCH}_{3}, \mathrm{HCOOCH}_{3}$, and 
$\mathrm{HOCH}_{2} \mathrm{CHO}$, via the surface-association reactions,

$$
\begin{aligned}
\mathrm{CH}_{3}+\mathrm{HCO} & \longrightarrow \mathrm{CH}_{3} \mathrm{CHO}, \\
\mathrm{CH}_{3} \mathrm{O}+\mathrm{CH}_{3} & \longrightarrow \mathrm{CH}_{3} \mathrm{OCH}_{3}, \\
\mathrm{CH}_{3} \mathrm{O}+\mathrm{HCO} & \longrightarrow \mathrm{HCOOCH}_{3}, \\
\mathrm{CH}_{2} \mathrm{OH}+\mathrm{HCO} & \longrightarrow \mathrm{HOCH}_{2} \mathrm{CHO},
\end{aligned}
$$

respectively. In this work, a ratio of 3:1:1 for pathways (9), (10), and (11) is assumed. This corresponds to the 'standard' case in the study of methyl formate formation in hot cores by Laas et al., (2011). 46

The faster diffusion rate of grain-surface radicals is apparent in the increased abundances of $\mathrm{HCOOH}, \mathrm{CH}_{3} \mathrm{CHO}, \mathrm{CH}_{3} \mathrm{OCH}_{3}, \mathrm{HCOOCH}_{3}$, and $\mathrm{HOCH}_{2} \mathrm{CHO}$ on the grain surface for the warm case. This leads to a higher abundance in the gas: grain-surface species formed on the grain are released to the gas via non-thermal desorption (see Section 3.2). Hence, the calculations for the warm case begin with significantly higher abundances of COMs more complex than methanol than for the cold case.

Table 1 Initial molecular fractional abundances and binding energies, $E_{B}$

\begin{tabular}{lccccc}
\hline & \multicolumn{2}{c}{ Cold } & \multicolumn{2}{c}{ Warm } & $E_{B}$ \\
Molecule & Gas & Ice & Gas & Ice & $(\mathrm{K})$ \\
\hline $\mathrm{CH}_{3} \mathrm{OH}$ & $9.8(-10)$ & $1.8(-06)$ & $1.3(-09)$ & $3.0(-06)$ & 5530 \\
$\mathrm{HCOOH}$ & $7.0(-11)$ & $2.8(-11)$ & $1.3(-09)$ & $1.7(-06)$ & 5570 \\
$\mathrm{CH}_{3} \mathrm{CHO}$ & $8.7(-12)$ & $9.8(-11)$ & $7.0(-11)$ & $1.2(-08)$ & 2780 \\
$\mathrm{CH}_{3} \mathrm{OCH}_{3}$ & $3.1(-14)$ & $1.5(-11)$ & $9.0(-11)$ & $8.1(-08)$ & 3680 \\
$\mathrm{HCOOCH}_{3}$ & $1.2(-15)$ & $2.3(-15)$ & $5.6(-11)$ & $1.2(-07)$ & 4100 \\
$\mathrm{HOCH}_{2} \mathrm{CHO}$ & $4.4(-21)$ & $1.8(-18)$ & $1.1(-11)$ & $6.6(-08)$ & 6680 \\
\hline \multicolumn{4}{l}{ Note: $a(b)=a \times 10^{b}$. Binding energies from Garrod et al., (2008). }
\end{tabular}

\section{Results}

\subsection{Physical structure}

The locations and paths of the streamlines over which the chemistry is calculated are shown in Figure 1 for the isolated case (top panel) and the irradiated case (bottom panel). The chemistry evolves along each streamline from outside to inside in the direction of increasing time (shown in the top axes of each panel). The disk scale height at large radii is larger for the irradiated disk due to the higher gas temperature in the disk midplane. The streamlines considered are those which are deep enough in the disk such that ices remain frozen out in the outer disk.

The number density (red lines) and gas temperature (blue lines) along each streamline as a function of disk radius is displayed in Figure 2. The integrated FUV flux (green lines) and X-ray flux (gold lines) as a function of radius are also shown in Figure 3. The data for the isolated disk are shown in the left-hand panel and those for the irradiated disk are shown in the right-hand panel.

12 | Faraday Discuss., [year], [vol], 1-36

This journal is @ The Royal Society of Chemistry [year] 
Fig. 1 Location and paths of streamlines along which the chemistry is calculated for the isolated disk (top panel) and irradiated disk (bottom panel). Time (shown on the top axes) increases from right to left.
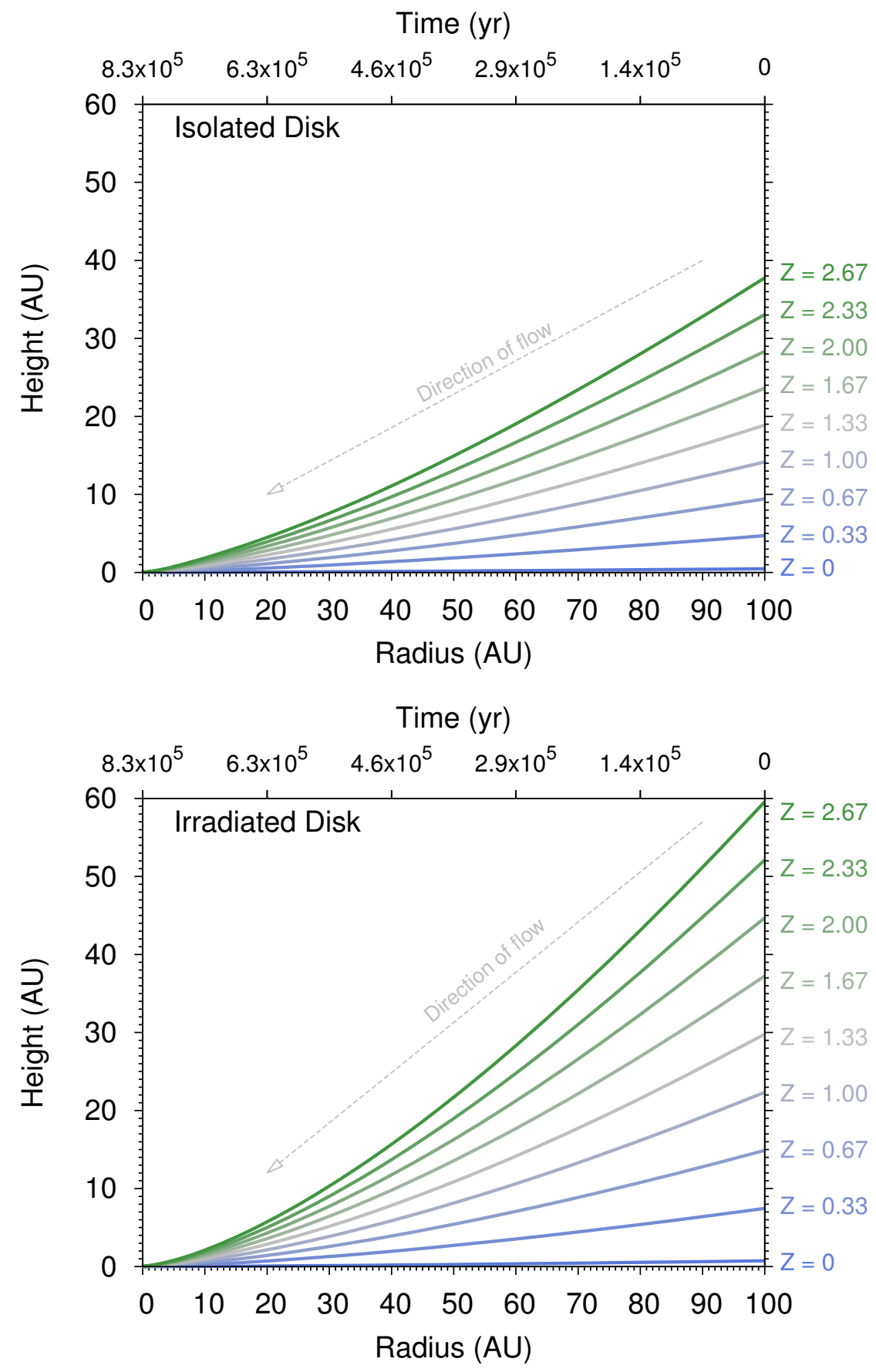

This journal is $\odot$ The Royal Society of Chemistry [year]

Faraday Discuss., [year], [vol], $1-36$ | 13 
The density structure in both disk models is similar along streamlines at the same scale height because the assumed surface density is the same in both models. The X-ray fluxes in both models are similar for the same reason i.e., the $\mathrm{X}$-rays 'see' a similar attenuating column of material. The temperature in the outer region ( $\gtrsim 10 \mathrm{AU}$ ) of the irradiated disk (right-hand panel) is significantly higher than for the isolated disk. This is due to the increased FUV flux from the nearby O-type star which is shown in Figure 3 . However, the temperature in the inner region $(\lesssim 1 \mathrm{AU})$ of the isolated disk is higher than that of the irradiated disk for the higher streamlines $(\gtrsim 2 H)$. This is because at small radii, the disk scale height for the isolated disk is slightly larger than for the irradiated disk; hence, the higher streamlines for the isolated disk flow through regions of higher temperature (and thus lower density). This is also demonstrated by the increased FUV flux for these streamlines (see Figure 3).

\subsection{Chemical evolution}

The fractional abundance (with respect to $\mathrm{H}$ nuclei density) of each species listed in Table 1 is shown in Figures 4 to 13 for both the isolated and irradiated disk models. We do not show the results for $\mathrm{CH}_{3} \mathrm{OCH}_{3}$ because the behaviour is the same as that for $\mathrm{HCOOCH}_{3}$. The blue and red lines in each plot represent the results for which the 'cold' and 'warm' sets of initial molecular abundances are assumed, respectively. There are several general trends. The ice mantle survives to larger scale heights in the isolated disk than in the irradiated disk $(Z \lesssim 2 H$ versus $Z \lesssim 0.67 H$ ). The stronger UV field in the outer regions of the irradiated disk efficiently strips the ice from the grain surface via photodesorption. Gas-phase COMs are subsequently destroyed by photodissociation. The results suggest gasphase COMs do not survive in the molecular layers of externally irradiated disks. The gas-phase COMs in both models reach their highest fractional abundance in the inner disk midplane. The origin of the gas-phase COMs is either thermal desorption from the ice mantle or gas-phase formation. The dominant process depends on the composition of the ice mantle entering the inner disk. The initial abundances (i.e., cold or warm) are not as critical for the isolated disk as for the irradiated disk. For those COMs which begin with a negligible abundance on the grain for the cold case, these species are formed efficiently on the grain surface under the conditions in the outer isolated disk (see, e.g., Figures 8, 10, and 12). On the other hand, for the irradiated disk, the initial composition is critical.

The specific behaviour and chemistry of the species predicted by reactions (12) to (15) are now discussed.

4.2.1 Methanol. In Figures 4 and 5 the fractional abundance of gas-phase (left-hand panel) and grain-surface (right-hand panel) methanol, $\mathrm{CH}_{3} \mathrm{OH}$, is shown as a function of disk radius for the isolated and irradiated disk models, respectively.

The methanol abundance is preserved along the accretion flow in the midplane in both models. At $\approx 2 \mathrm{AU}$, the temperature is sufficiently high for methanol ice to thermally desorb from the grain surface into the gas phase. This transition region is known as the snowline and resides at the same radius for both models. This is because the heating in the inner disk is dominated by the central star and viscous dissipation rather than external irradiation. The abundance reached

14 | Faraday Discuss., [year], [vol], 1-36

This journal is @ The Royal Society of Chemistry [year] 
Fig. 2 Number density (blue lines) and gas temperature (red lines) along each streamline as a function of radius for the isolated disk (left-hand panel) and irradiated disk (right-hand panel). Time (shown on the top axes) increases from right to left.
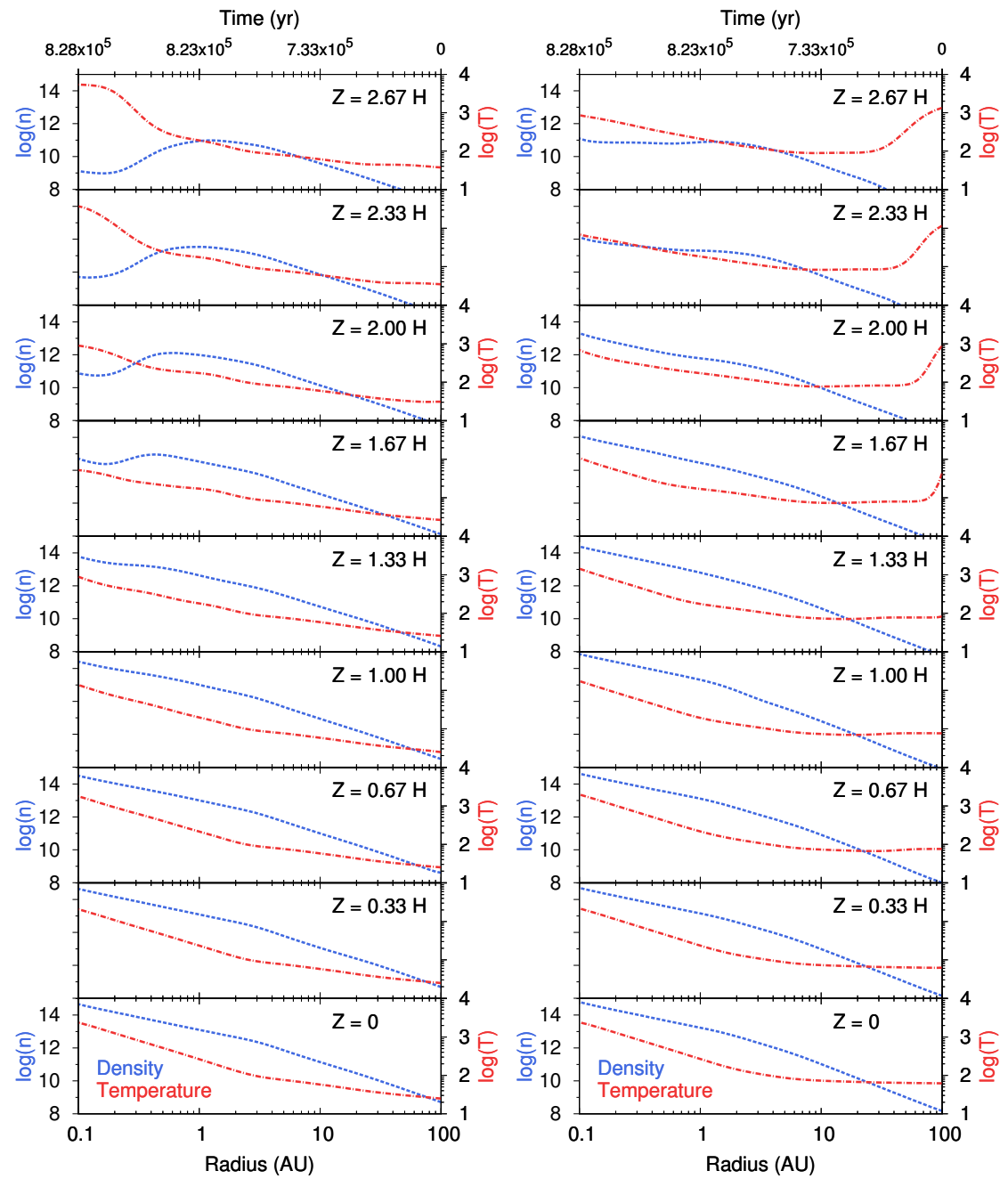
Fig. 3 FUV flux (green lines) and X-ray flux (gold lines) along each streamline as a function of radius for the isolated disk (right-hand panel) and irradiated disk (left-hand panel). The units are erg $\mathrm{cm}^{-2} \mathrm{~s}^{-1}$. Time (shown on the top axes) increases from right to left.
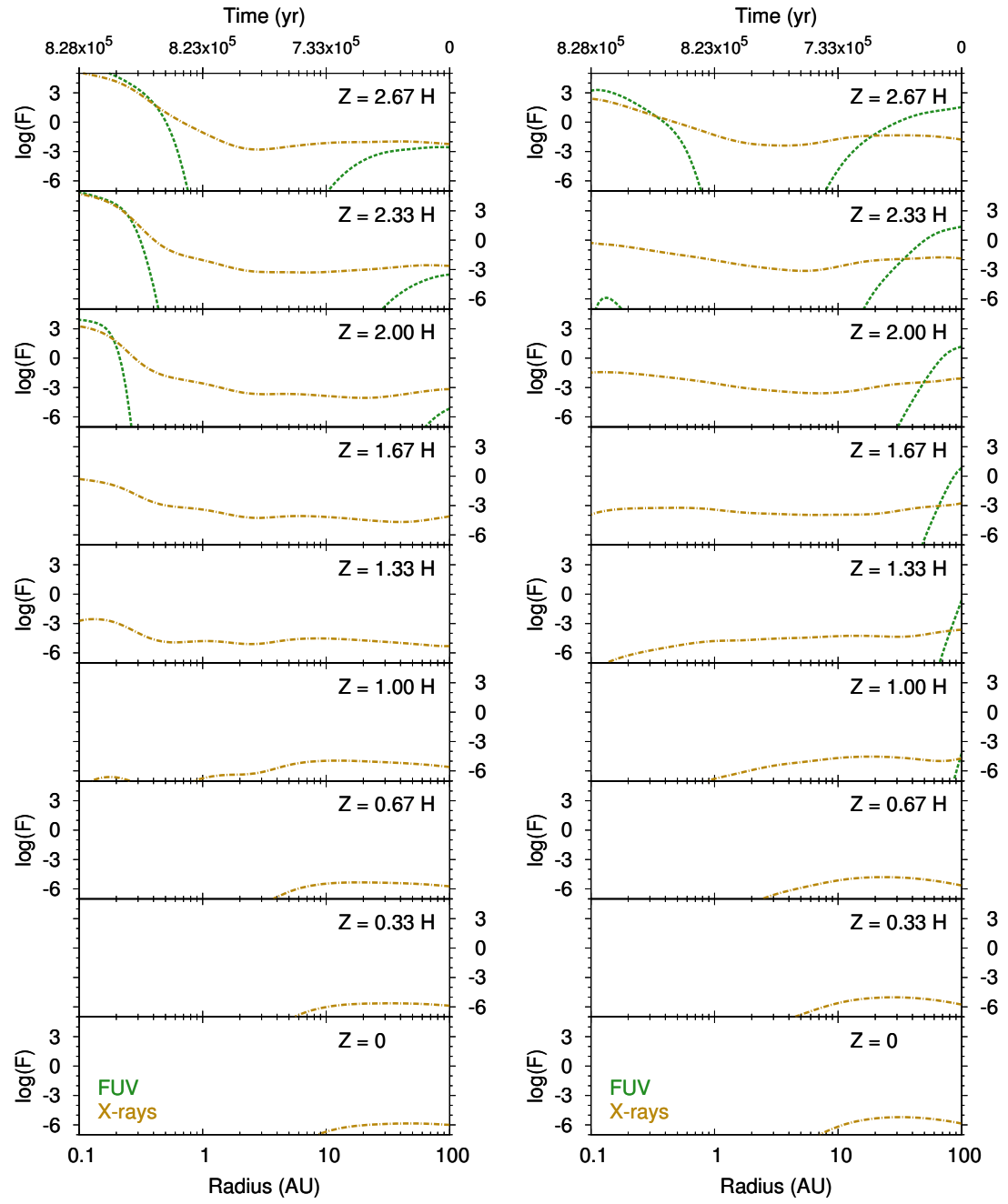

16 | Faraday Discuss., [year], [vol], $1-\sqrt{36}$ 
in the gas phase is the same as that for the ice injected into both models. This suggests that the methanol entering the planet- and comet-forming region in both irradiated and isolated protoplanetary disks has an interstellar origin.

At higher scale heights in the isolated disk, methanol is photodesorbed into the gas phase reaching a fractional abundance $\sim 10^{-9}$ with respect to number density at radii $\gtrsim 50 \mathrm{AU}$. This photodesorbed layer is not present in the irradiated case. Whether gas-phase molecules survive in the photodesorbed layer requires a delicate balance between photodesorption and photodissociation in the molecular layer of the disk. The results suggest that gas-phase methanol may be observable in isolated protoplanetary disks as was found in the static model. 19

There are only minor differences between the cold and warm sets of abundances for both disk models because methanol ice reaches similar fractional abundances in the cold and warm cloud models $\left(\approx 10^{-6}\right)$.

4.2.2 Formic acid. In Figures 6 and 7 the fractional abundance of gas-phase (left-hand panel) and grain-surface (right-hand panel) formic acid, $\mathrm{HCOOH}$, is shown as a function of disk radius for the isolated and irradiated disk models, respectively. The story for formic acid is different from that for methanol. Formic acid is postulated to possess several routes to formation on grain surfaces at low temperatures, including the radical-radical association reaction,

$$
\mathrm{HCO}+\mathrm{OH} \longrightarrow \mathrm{HCOOH} .
$$

$\mathrm{HCOOH}$ has also been postulated to form via hydrogenation of the 'HOCO' complex ${ }^{93}$ i.e.,

$$
\begin{gathered}
\mathrm{CO}+\mathrm{OH} \longrightarrow \mathrm{HOCO}, \\
\mathrm{HOCO}+\mathrm{H} \longrightarrow \mathrm{HCOOH} .
\end{gathered}
$$

Öberg et al. studied the formation of $\mathrm{HCOOH}$ via the first reaction during the irradiation of pure methanol ice. Upper limits only were determined due to the difficulty uniquely identifying $\mathrm{HCOOH}$ bands in the RAIRS (reflection-absorption infrared spectroscopy) spectrum. ${ }^{50}$ Ioppolo et al. later studied the formation of $\mathrm{HCOOH}$ via the second pathway and determined it was efficient at low temperatures $\lesssim 20 \mathrm{~K} \cdot{ }^{93}$ Both pathways are included in the network used here; however, the reaction, $\mathrm{CO}+\mathrm{OH} \rightarrow \mathrm{HOCO}$, has a large reaction barrier, $\gtrsim 1500 \mathrm{~K}$, originating from studies of the gas-phase reaction potential energy surface. ${ }^{94}$ Because thermal grain-surface chemistry is used, this reaction is slow at low temperatures and $\mathrm{HCOOH}$ is not efficiently formed at $\sim 10 \mathrm{~K}$. As a consequence, the distribution of $\mathrm{HCOOH}$ along the accretion flow is sensitive to the assumed initial abundance.

In the isolated disk, the abundance of $\mathrm{HCOOH}$ ice and gas for the warm case is around two orders of magnitude higher than for the cold case. A comparison with the initial abundances shows that grain-surface $\mathrm{HCOOH}$ is formed in the outer disk for the cold case in both models albeit reaching a lower abundance than that achieved for the warm case. In the isolated disk, as found for methanol, the formic acid fractional abundance, $\sim 10^{-7}-10^{-6}$, is preserved along the accretion flow. The snowline for $\mathrm{HCOOH}$ also resides at a similar radius to that for methanol $(\approx 2 \mathrm{AU})$ reflecting their similar binding energies (see Table 1). At

This journal is @ The Royal Society of Chemistry [year]

Faraday Discuss., [year], [vol], 1-36 | 17 
Fig. 4 Fractional abundance (with respect to number density) of $\mathrm{CH}_{3} \mathrm{OH}$ gas and ice along each streamline as a function of disk radius for the isolated disk model. The blue lines show results from the model using the 'cold' $(10 \mathrm{~K})$ set of initial abundances and the red lines from the model using the 'warm' $(30 \mathrm{~K})$ set of initial abundances (see, Table 1 .

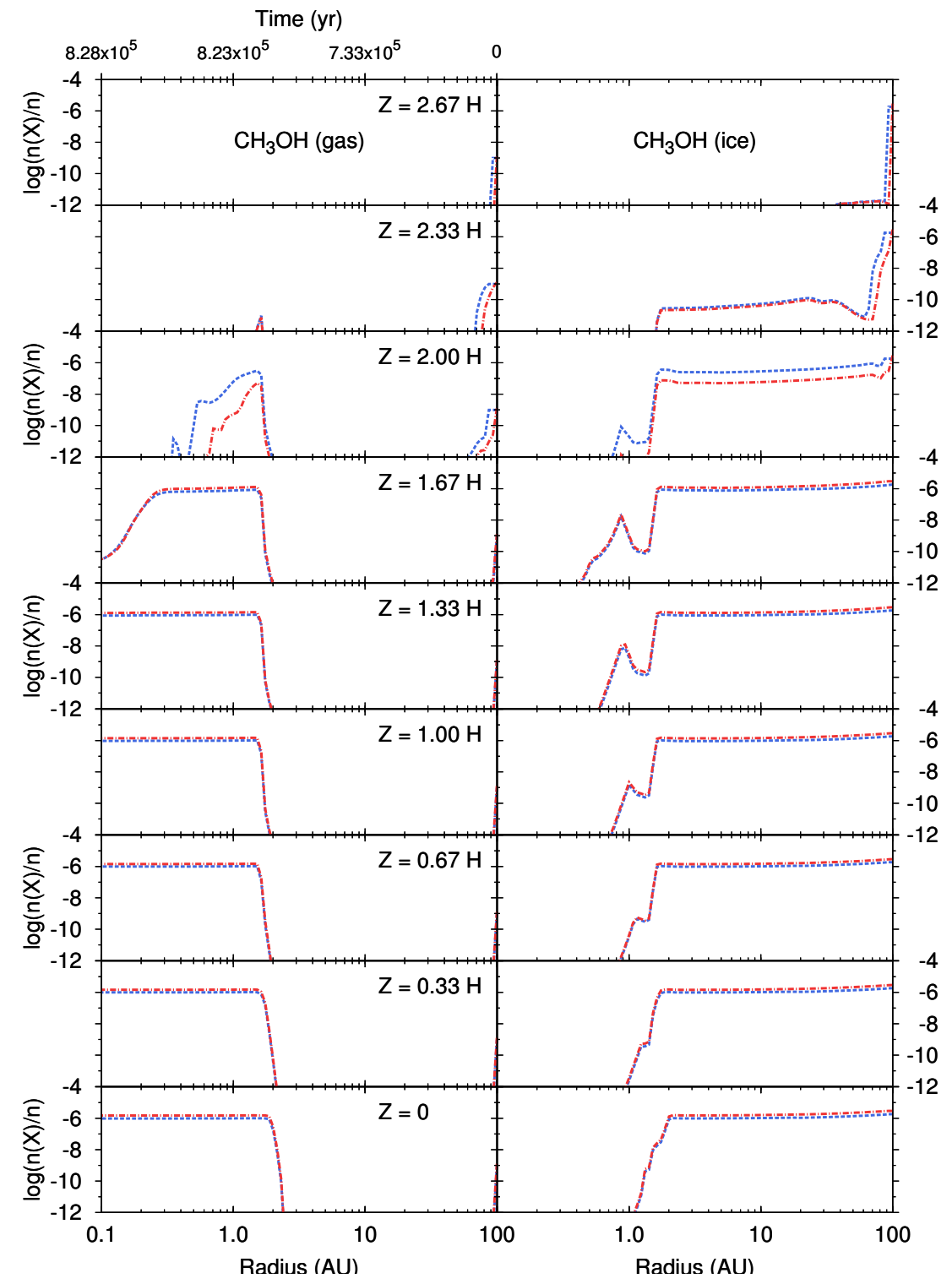

18 | Faraday Discuss., [year], [vol], 1-36

This journal is @ The Royal Society of Chemistry [year] 
Fig. 5 Fractional abundance (with respect to number density) of $\mathrm{CH}_{3} \mathrm{OH}$ gas and ice along each streamline as a function of disk radius for the irradiated disk model. The blue lines show results from the model using the 'cold' $(10 \mathrm{~K})$ set of initial abundances and the red lines from the model using the 'warm' $(30 \mathrm{~K})$ set of initial abundances (see, Table 1 ).

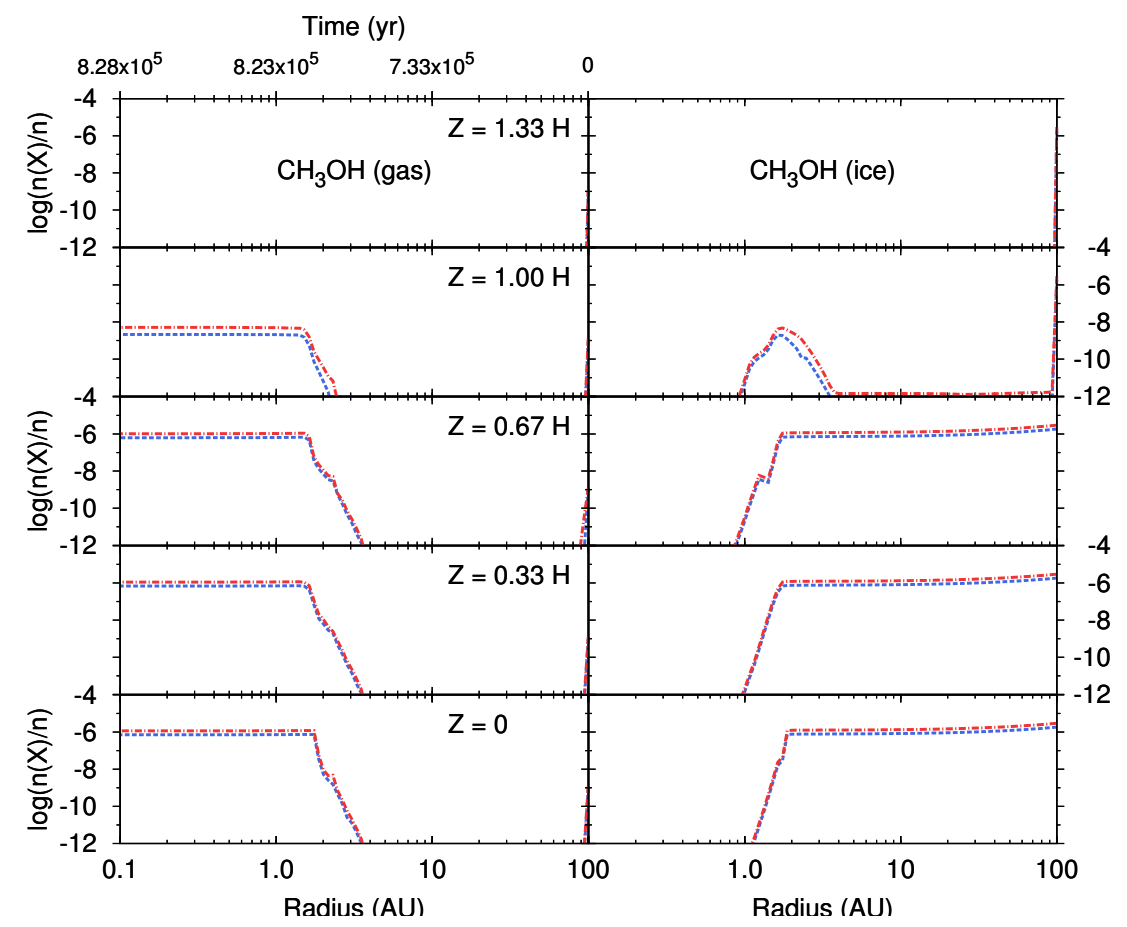


higher scale heights in the isolated disk, formic acid is photodesorbed reaching a gas-phase fractional abundance $\sim 10^{-9}$. Formic acid ice transported into the inner regions of isolated protoplanetary disks may have an interstellar origin provided the initial abundance entering the disk is sufficiently high. $\mathrm{HCOOH}$ ice has been observed in the envelopes of several low-mass protostars with abundances $\sim 1-5 \%$ of the water ice abundance. 95

In the irradiated disk, the gas-phase formic acid abundance in the inner region of the disk does not reflect that injected into the outer disk. The higher temperature $(>60 \mathrm{~K})$ in the midplane helps thermally process the ice such that the cold and warm models converge at a radius of $\approx 5 \mathrm{AU}$, i.e., outside the snowline at $\approx 2 \mathrm{AU}$. This thermal processing leads to a drop in the fractional abundance of formic acid ice from $\sim 10^{-8}-10^{-6}$ to $10^{-10}$ at $\approx 5$ AU. Hence, the thermally desorbed formic acid inside of the snowline reaches a peak fractional abundance $\sim 10^{-10}$. Molecules which rely on radical-radical association reactions are sensitive to the higher temperature because radicals generally possess a lower binding energy to the grain surface than molecules, e.g., the binding energy assumed here for $\mathrm{OH}$ and $\mathrm{HCO}$ are $2850 \mathrm{~K}$ and $1600 \mathrm{~K}$, respectively. Whether a molecule can form efficiently via this process relies on a sufficient supply of precursor radicals on the grain surface. At higher temperatures, the thermal desorption of radicals begins to compete with the thermal grain-surface association rates. For the case of formic acid in the irradiated disk midplane, this occurs at $\approx 80 \mathrm{~K}$ (at $\approx 5 \mathrm{AU})$.

4.2.3 Acetaldehyde. In Figures 8 and 9 the fractional abundance of gasphase (left-hand panel) and grain-surface (right-hand panel) acetaldehyde, $\mathrm{CH}_{3} \mathrm{CHO}$, is shown as a function of disk radius for the isolated and irradiated disk models, respectively.

In contrast to formic acid, acetaldehyde is not sensitive to the assumed initial abundances. In the isolated disk for the cold case, $\mathrm{CH}_{3} \mathrm{CHO}$ forms efficiently in the outer disk midplane reaching a similar ice fractional abundance as for the warm case, $\sim 10^{-7}$, for radii $\gtrsim 10 \mathrm{AU}$. Acetaldehyde ice can from on the grain via the association of the $\mathrm{CH}_{3}$ and $\mathrm{HCO}$ radicals. This reaction is barrierless and so can proceed at the low temperatures in the outer regions of the isolated disk. The snowline for acetaldehyde resides at $\approx 10 \mathrm{AU}$ reflecting its lower binding energy $(2780 \mathrm{~K})$. There is a narrow thermally desorbed region of gas-phase acetaldehyde at $\approx 10 \mathrm{AU}$. Within this radius, the temperature is sufficiently high for efficient thermal desorption of both the $\mathrm{CH}_{3}$ and $\mathrm{HCO}$ radicals so that the radicalradical association formation pathway is no longer effective. Acetaldehyde can also form via hydrogenation of $\mathrm{CH}_{3} \mathrm{CO}$ which itself forms via the association of $\mathrm{CH}_{3}$ and $\mathrm{CO}$; however, this reaction has a large reaction barrier $(\approx 3500 \mathrm{~K})$ and $\mathrm{CO}$ is also a volatile species with a binding energy $1150 \mathrm{~K}$. The origin of gas-phase acetaldehyde in the inner disk midplane $(\lesssim 2 \mathrm{AU})$ is thus gas-phase chemistry induced by the thermal desorption of strongly bound COMs, for example, $\mathrm{C}_{2} \mathrm{H}_{5} \mathrm{OH}$. Gas-phase acetaldehyde forms primarily via dissociative electron recombination of the protonated form, $\mathrm{CH}_{3} \mathrm{CH}_{2} \mathrm{O}^{+}$, which can form via reaction of cations with $\mathrm{C}_{2} \mathrm{H}_{5} \mathrm{OH}$.

Acetaldehyde ice in the outer region of the disk may have an interstellar origin, again, if the injected abundance is sufficiently high. There are no reported detections of acetaldehyde ice in molecular clouds or protostellar envelopes. However, gas-phase acetaldehyde has been detected towards several dark clouds and 
Fig. 6 Fractional abundance (with respect to number density) of $\mathrm{HCOOH}$ gas and ice along each streamline as a function of disk radius for the isolated disk model. The blue lines show results from the model using the 'cold' $(10 \mathrm{~K})$ set of initial abundances and the red lines from the model using the 'warm' $(30 \mathrm{~K})$ set of initial abundances (see, Table 1 .

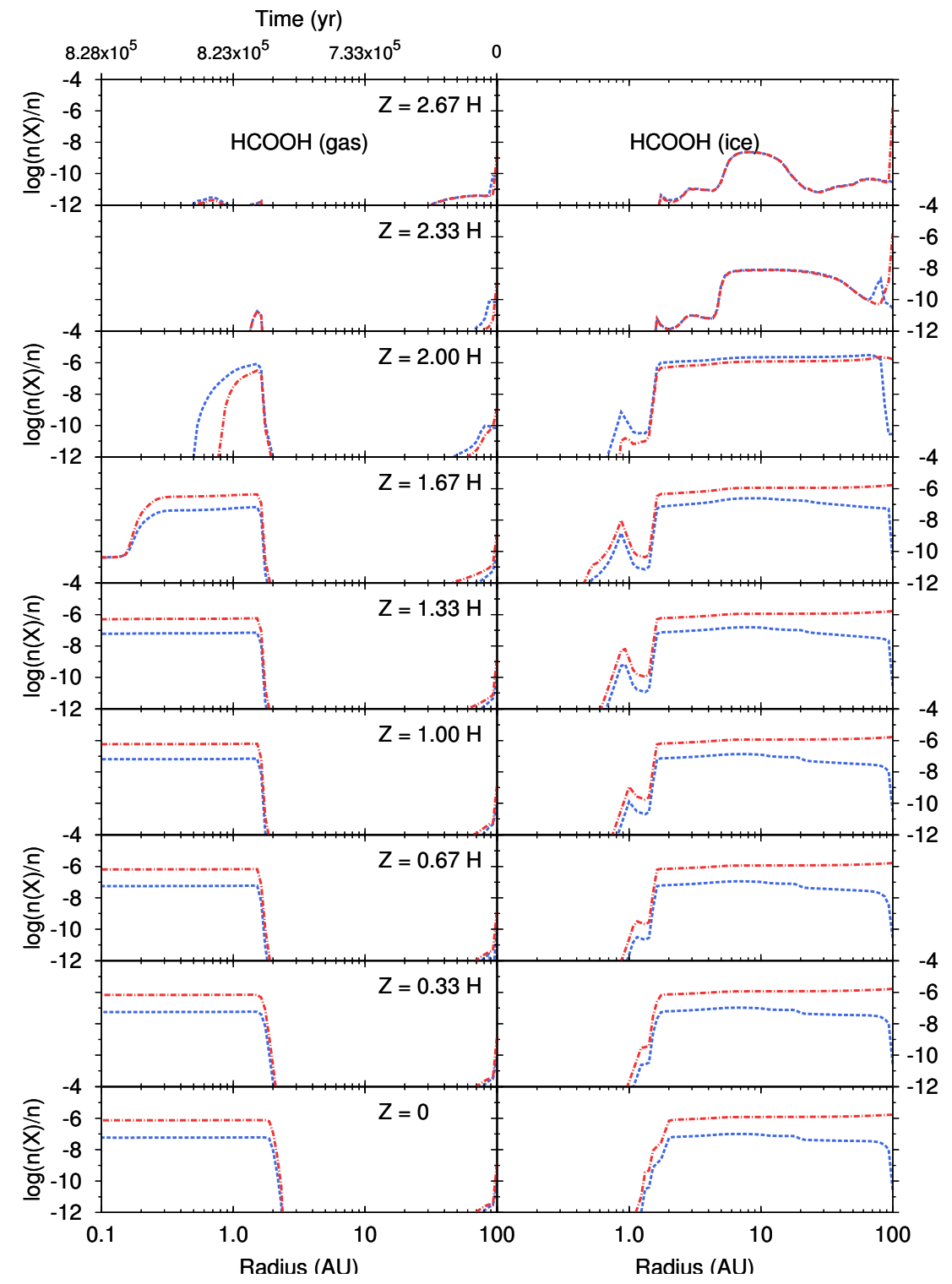


Fig. 7 Fractional abundance (with respect to number density) of $\mathrm{HCOOH}$ gas and ice along each streamline as a function of disk radius for the irradiated disk model. The blue lines show results from the model using the 'cold' $(10 \mathrm{~K})$ set of initial abundances and the red lines from the model using the 'warm' $(30 \mathrm{~K})$ set of initial abundances (see, Table 1 ).

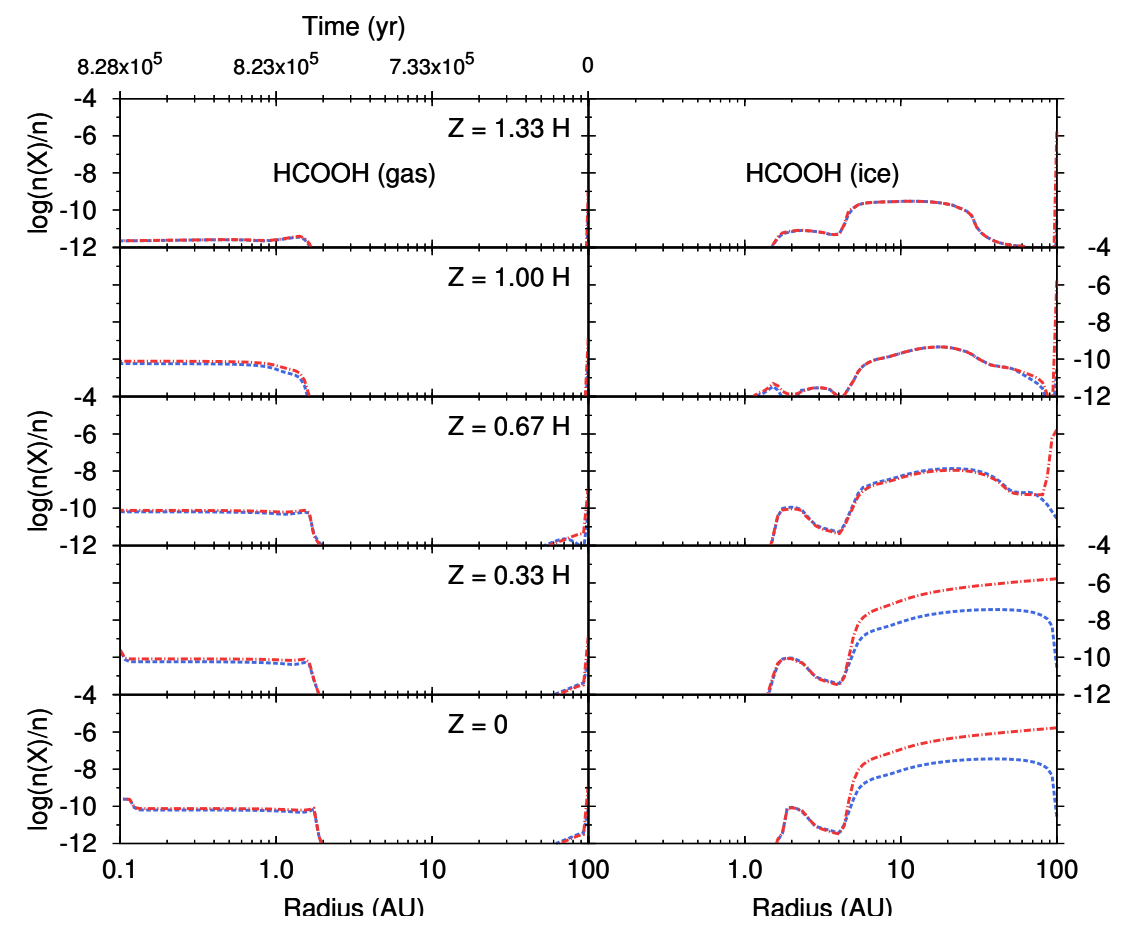

22 | Faraday Discuss., [year], [vol], 1-36

This journal is $\odot$ The Royal Society of Chemistry [year] 
prestellar cores with a fractional abundance $\sim 10^{-11}-10^{-10}$ with respect to the $\mathrm{H}_{2}$ gas density. 96 The relatively constant abundance across sources suggests the source of $\mathrm{CH}_{3} \mathrm{CHO}$ is grain-surface formation followed by non-thermal desorption into the gas phase.

In the irradiated disk, the temperature is sufficiently high to thermally desorb $\mathrm{CH}_{3} \mathrm{CHO}$ (and its precursor radicals) such that the molecule survives neither on the grain, nor in the gas. $\mathrm{CH}_{3} \mathrm{CHO}$ molecules destroyed in the gas phase cannot be replenished via grain-surface chemistry due to the volatile nature of $\mathrm{CH}_{3}$ and $\mathrm{HCO}$. This result suggests $\mathrm{CH}_{3} \mathrm{CHO}$ ice which is formed in comets within externally irradiated disks may have a secondary origin and is not pristine. The origin of gas-phase acetaldehyde in the inner disk is gas-phase formation following the thermal desorption of strongly bound COMs. Thus the fractional abundance of gas-phase acetaldehyde in the inner regions of the isolated disk and the irradiated disk are similar, $\sim 10^{-9}$.

4.2.4 Methyl formate. In Figures 10 and 11 the fractional abundance of gas-phase (left-hand panel) and grain-surface (right-hand panel) methyl formate, $\mathrm{HCOOCH}_{3}$, is shown as a function of disk radius for the isolated and irradiated disk models, respectively.

For the isolated disk, the abundance of methyl formate is not sensitive to the assumed initial abundances: methyl formate is efficiently formed in the outer disk via the association of the $\mathrm{CH}_{3} \mathrm{O}$ and $\mathrm{HCO}$ radicals, reaching a peak fractional abundance, $\sim 10^{-7}$. This reaction is barrierless and can proceed at the temperatures in the outer disk, $\approx 20 \mathrm{~K}$. This agrees with the result found for the static model. 19 The snowline for methyl formate lies slightly beyond the methanol snowline, between 2 and 3 AU. This reflects the slightly lower binding energy assumed for methyl formate $(4100 \mathrm{~K})$. The abundance of methyl formate is preserved in the inner disk region. The origin of methyl formate in the planetand comet-forming regions of isolated disks may have an interstellar origin depending on the initial abundance entering the disk. Gas-phase methyl formate has been observed in dark clouds and prestellar cores with a fractional abundance similar to that for acetaldehyde $\sim 10^{-11}-10^{-10} .96$

In the irradiated disk model, the abundance of methyl formate ice is sensitive to the assumed initial abundances. For the cold case, methyl formate ice reaches a peak fractional abundance $\sim 10^{-10}$ contrasted with $\sim 10^{-7}$ for the warm case. Again, the higher temperature in the irradiated disk leads to efficient desorption of the necessary precursor radicals, $\mathrm{CH}_{3} \mathrm{O}$ and $\mathrm{HCO}$, which have assumed binding energies, $2500 \mathrm{~K}$ and $1600 \mathrm{~K}$, respectively. Methyl formate thermally desorbs at a radius $\approx 5 \mathrm{AU}$ whereby it is destroyed by gas-phase chemistry. Within $\approx 2 \mathrm{AU}$, gas-phase methyl formate is replenished via gas-phase chemistry induced by the thermal desorption of strongly bound COMs. Methyl formate can form in the gas via the dissociative recombination of the protonated form of the molecule, $\mathrm{HCOOCH}_{4}^{+}$. This species, in turn, can form via the following ionneutral reactions,

$$
\begin{aligned}
& \mathrm{CH}_{3} \mathrm{OH}_{2}^{+}+\mathrm{HCOOH} \longrightarrow \mathrm{HCOOCH}_{4}^{+}+\mathrm{H}_{2} \mathrm{O} \\
& \mathrm{HCOOH}_{2}^{+}+\mathrm{CH}_{3} \mathrm{OH} \longrightarrow \mathrm{HCOOCH}_{4}^{+}+\mathrm{H}_{2} \mathrm{O} .
\end{aligned}
$$

Thus, the formation of gas-phase methyl formate is triggered by the thermal des- 
Fig. 8 Fractional abundance (with respect to number density) of $\mathrm{CH}_{3} \mathrm{CHO}$ gas and ice along each streamline as a function of disk radius for the isolated disk model. The blue lines show results from the model using the 'cold' $(10 \mathrm{~K})$ set of initial abundances and the red lines from the model using the 'warm' $(30 \mathrm{~K})$ set of initial abundances (see, Table 1 .

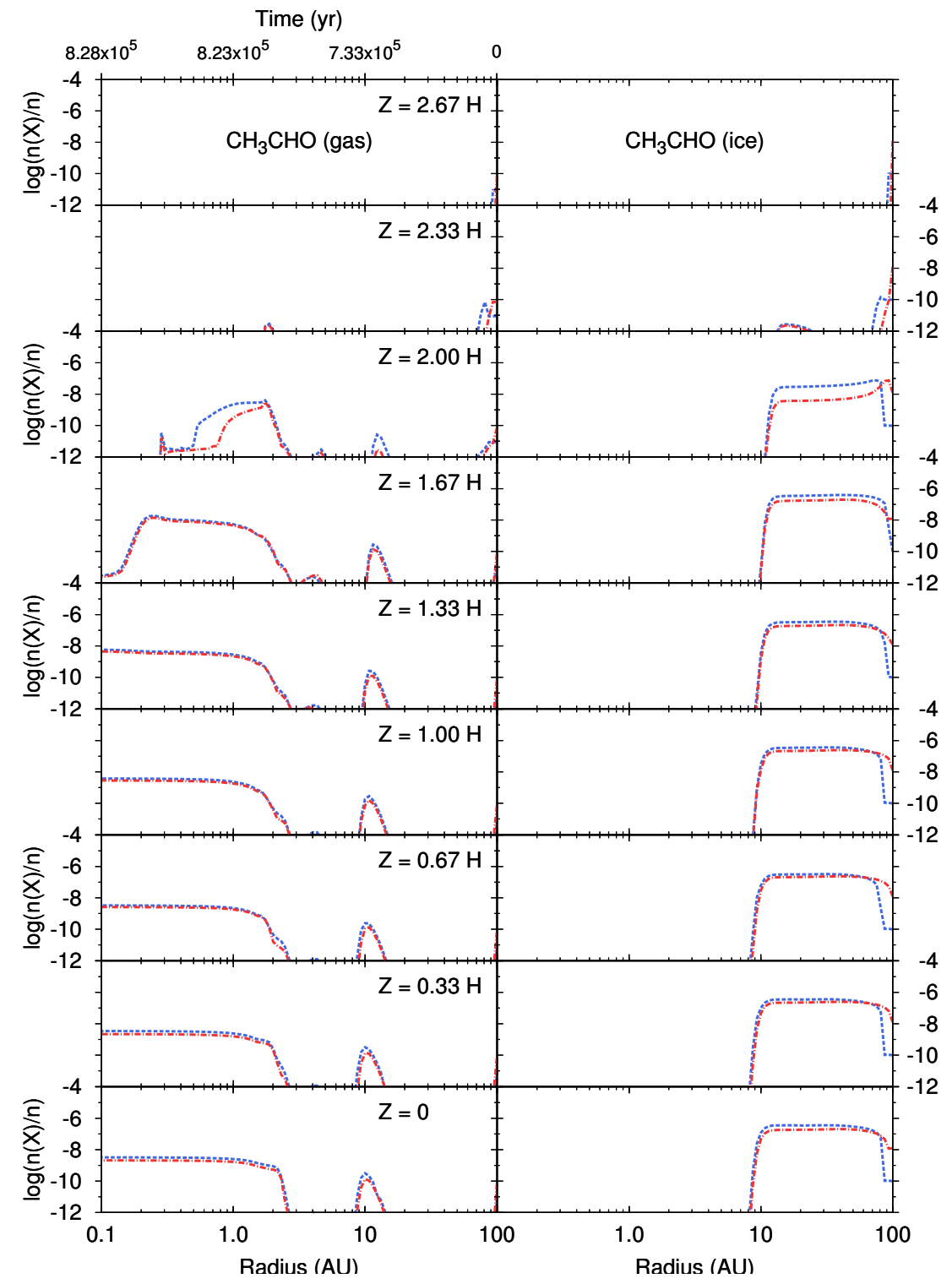

24 | Faraday Discuss., [year], [vol], 1-36

This journal is @ The Royal Society of Chemistry [year] 
Fig. 9 Fractional abundance (with respect to number density) of $\mathrm{CH}_{3} \mathrm{CHO}$ gas and ice along each streamline as a function of disk radius for the irradiated disk model. The blue lines show results from the model using the 'cold' $(10 \mathrm{~K})$ set of initial abundances and the red lines from the model using the 'warm' $(30 \mathrm{~K})$ set of initial abundances (see, Table 1 ).

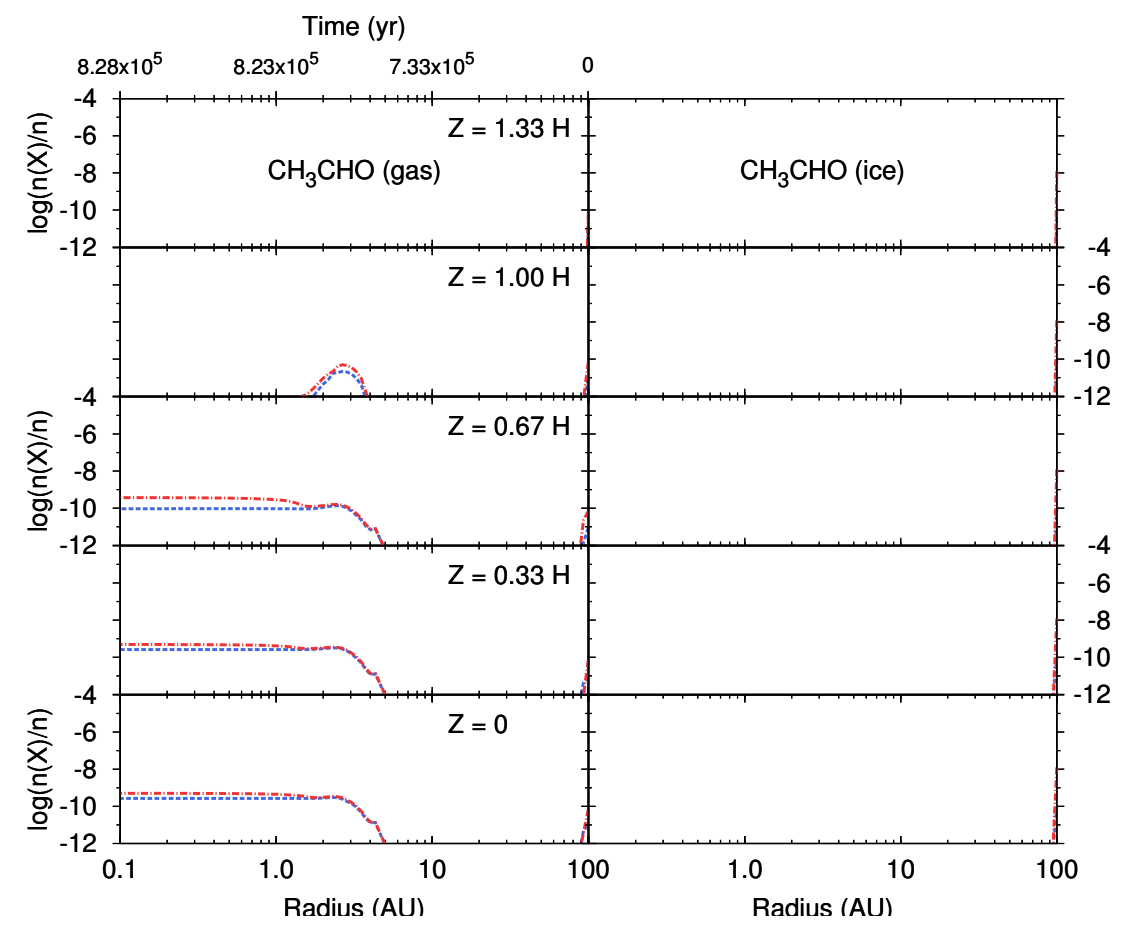


orption of $\mathrm{CH}_{3} \mathrm{OH}$ and $\mathrm{HCOOH}$ ice within $\approx 2 \mathrm{AU}$. The peak fractional abundance attained is $\sim 10^{-9}$ compared with $\sim 10^{-7}$ for the isolated case. This result correlates well with previous work which concluded gas-phase formation of methyl formate contributes, at most, to $\sim 1 \%$ of the total gas-phase abundance in hot cores. $\stackrel{46}{\underline{46}}$

The scenario described here for methyl formate, $\mathrm{HCOOCH}_{3}$, is similar to that for dimethyl ether, $\mathrm{CH}_{3} \mathrm{OCH}_{3}$.

4.2.5 Glycolaldehyde. In Figures 12 and 13 the fractional abundance of gas-phase (left-hand panel) and grain-surface (right-hand panel) glycolaldehyde, $\mathrm{HOCH}_{2} \mathrm{CHO}$, is shown as a function of disk radius for the isolated and irradiated disk models, respectively. Glycolaldehyde is an interesting complex molecule because it is the simplest molecule which possesses both an aldehyde and a hydroxyl group. It has also recently been detected in the gas phase towards the low-mass protostar, IRAS-16293 with a fractional abundance, $\sim 10^{-8}$ with respect to $\mathrm{H}_{2} \cdot 99$

Similar to methyl formate, glycolaldehyde is not sensitive to assumed initial abundances in the outer regions of the isolated disk and can form efficiently via the barrierless reaction between $\mathrm{CH}_{2} \mathrm{OH}$ and $\mathrm{HCO}$. Glycolaldehyde thermally desorbs within a radius of $\approx 2 \mathrm{AU}$ and exists in the gas phase in a narrow region between $\approx 1$ and $2 \mathrm{AU}$. Within this region, the conditions are such that grainsurface reformation cannot compete with gas-phase destruction on the timescales of the accretion flow; however, it is possible that the gas-phase chemical network is incomplete for larger COMs such as glycolaldehyde.

The glycolaldehyde ice abundances in the outer region of the irradiated disk are very sensitive to the assumed initial abundances, again, requiring an appreciable abundance of precursor radicals on the grain. Once the glycolaldehyde desorbs, it is efficiently destroyed in the gas phase.

\subsection{Comparison with previous disk models}

The influence of the accretion flow (or advection) on the gas-grain balance in protoplanetary disks has been investigated previously. ${ }^{21}-24$ Aikawa et al. computed the chemistry along streamlines from the outer disk $(\approx 400-600 \mathrm{AU})$ to the inner disk $(\approx 10-70 \mathrm{AU})$ using a method similar to that outlined here. Ilgner et al. and Heinzeller et al. later investigated the chemical evolution of parcels of gas moving inwards along the accretion flow along multiple trajectories to build twodimensional 'snapshots' of the chemical structure of the planet-forming region of the disk $(\lesssim 10 \mathrm{AU})$. In these works grain-surface chemistry was not included which restricted the discussion to small, simple species. Nomura et al. considered the chemistry and transport of COMs in a protoplanetary disk for the first time. However, as in previous works, a grain-surface network was not explicitly included and the calculations followed the subsequent gas-phase chemistry upon thermal desorption of the parent COMs assumed to be already present in the ice mantle. The discussion was also restricted to the inner planet-forming zone $(\lesssim 12 \mathrm{AU}$ ). Hence, the work presented here is the first investigation of grainsurface chemistry and the subsequent formation and destruction of COMs along the accretion flow in a protoplanetary disk.

26 | Faraday Discuss., [year], [vol], 1-36

This journal is @ The Royal Society of Chemistry [year] 
Fig. 10 Fractional abundance (with respect to number density) of $\mathrm{HCOOCH}_{3}$ gas and ice along each streamline as a function of disk radius for the isolated disk model. The blue lines show results from the model using the 'cold' (10 K) set of initial abundances and the red lines from the model using the 'warm' $(30 \mathrm{~K})$ set of initial abundances (see, Table 11.

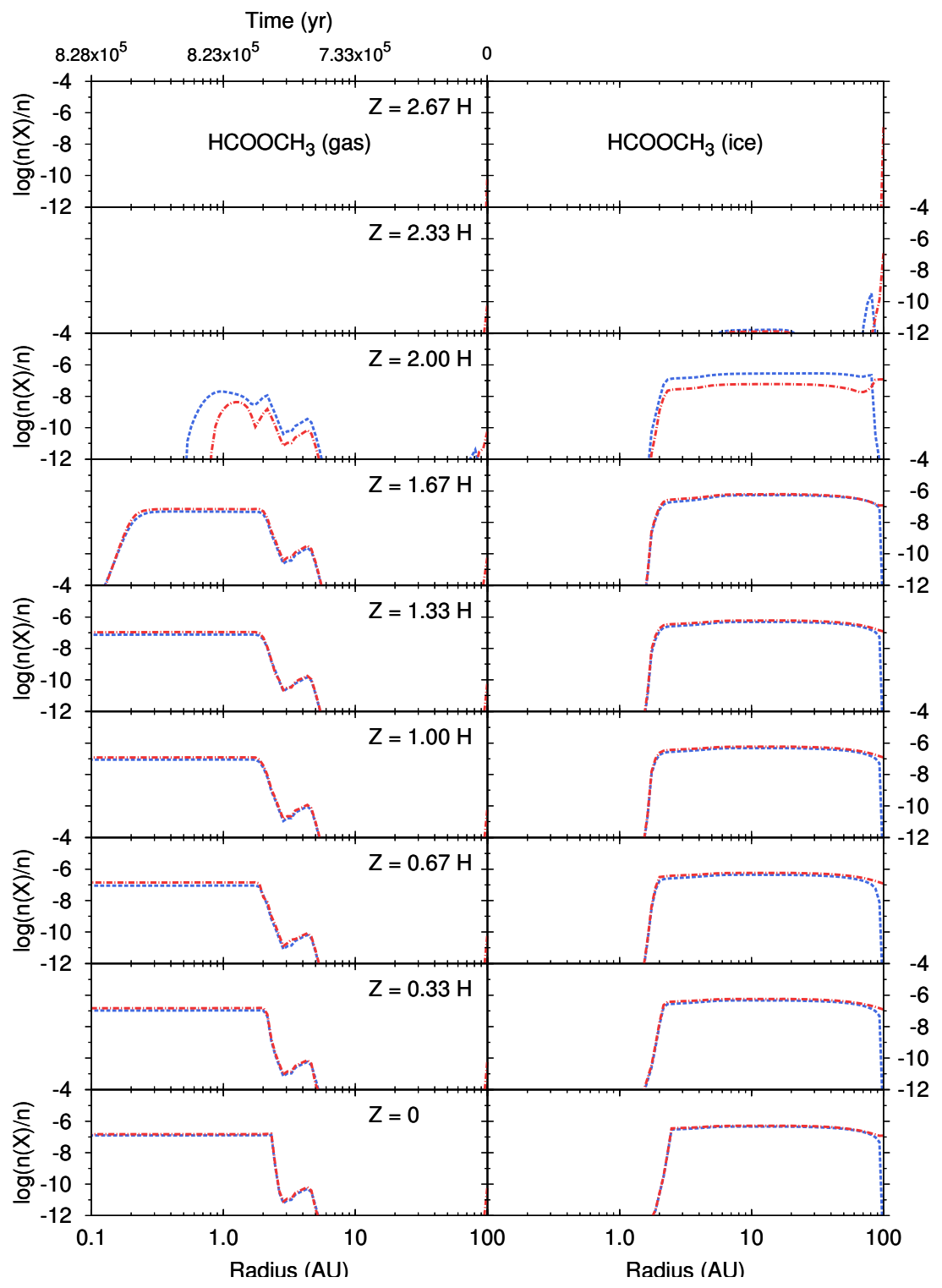


Fig. 11 Fractional abundance (with respect to number density) of $\mathrm{HCOOCH}_{3}$ gas and ice along each streamline as a function of disk radius for the irradiated disk model. The blue lines show results from the model using the 'cold' $(10 \mathrm{~K})$ set of initial abundances and the red lines from the model using the 'warm' $(30 \mathrm{~K})$ set of initial abundances (see, Table 1).

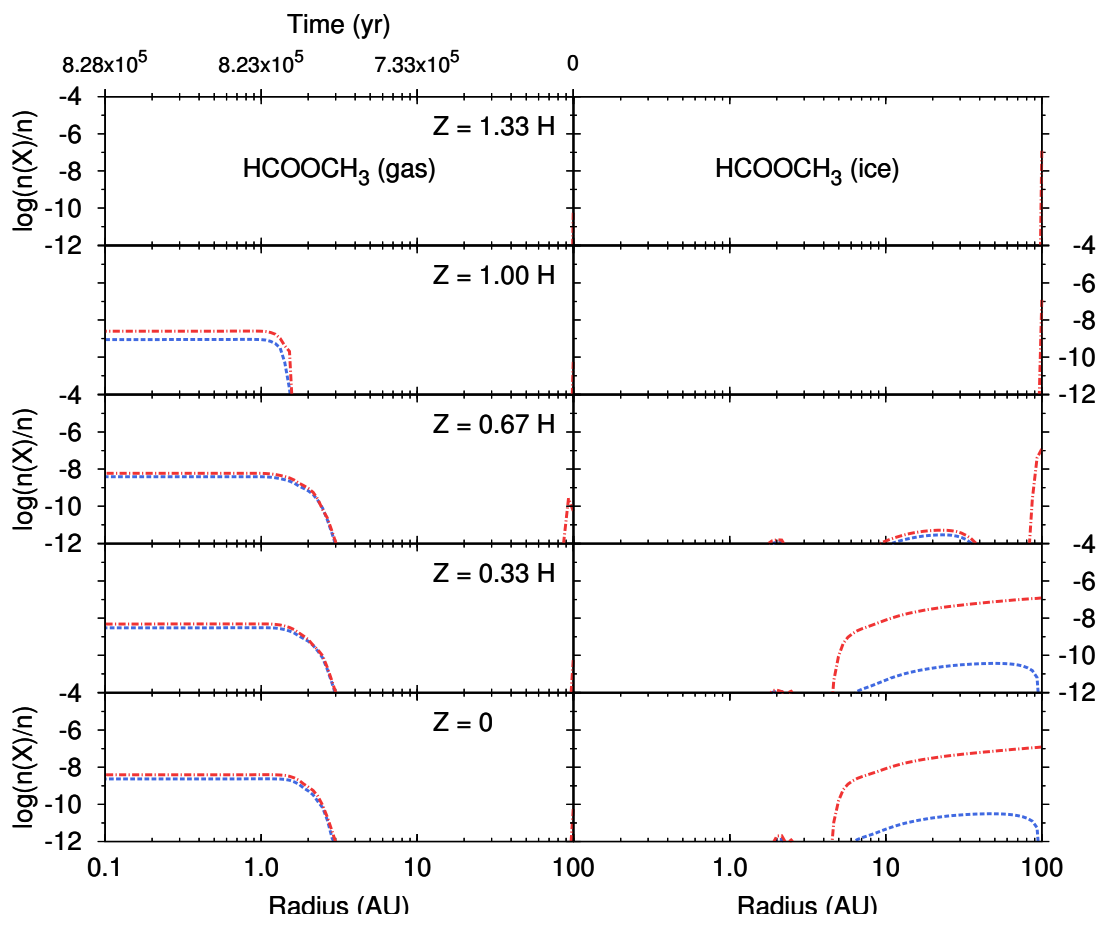

28 | Faraday Discuss., [year], [vol], $1-36$

This journal is @ The Royal Society of Chemistry [year] 
Fig. 12 Fractional abundance (with respect to number density) of $\mathrm{HOCH}_{2} \mathrm{CHO}$ gas and ice along each streamline as a function of disk radius for the isolated disk model. The blue lines show results from the model using the 'cold' $(10 \mathrm{~K})$ set of initial abundances and the red lines from the model using the 'warm' $(30 \mathrm{~K})$ set of initial abundances (see, Table 11.

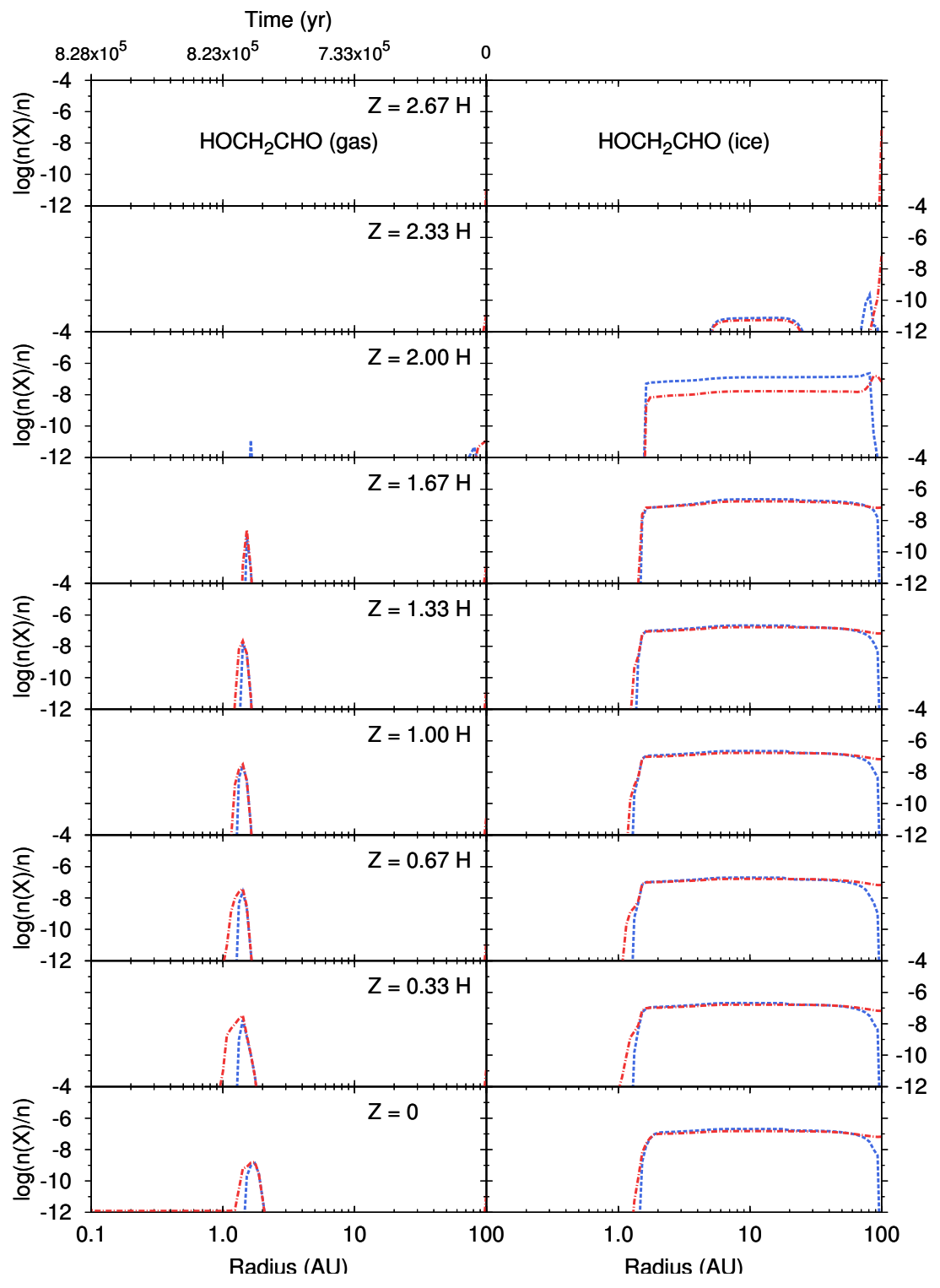


Fig. 13 Fractional abundance (with respect to number density) of $\mathrm{HOCH}_{2} \mathrm{CHO}$ gas and ice along each streamline as a function of disk radius for the irradiated disk model. The blue lines show results from the model using the 'cold' $(10 \mathrm{~K})$ set of initial abundances and the red lines from the model using the 'warm' $(30 \mathrm{~K})$ set of initial abundances (see, Table 1).

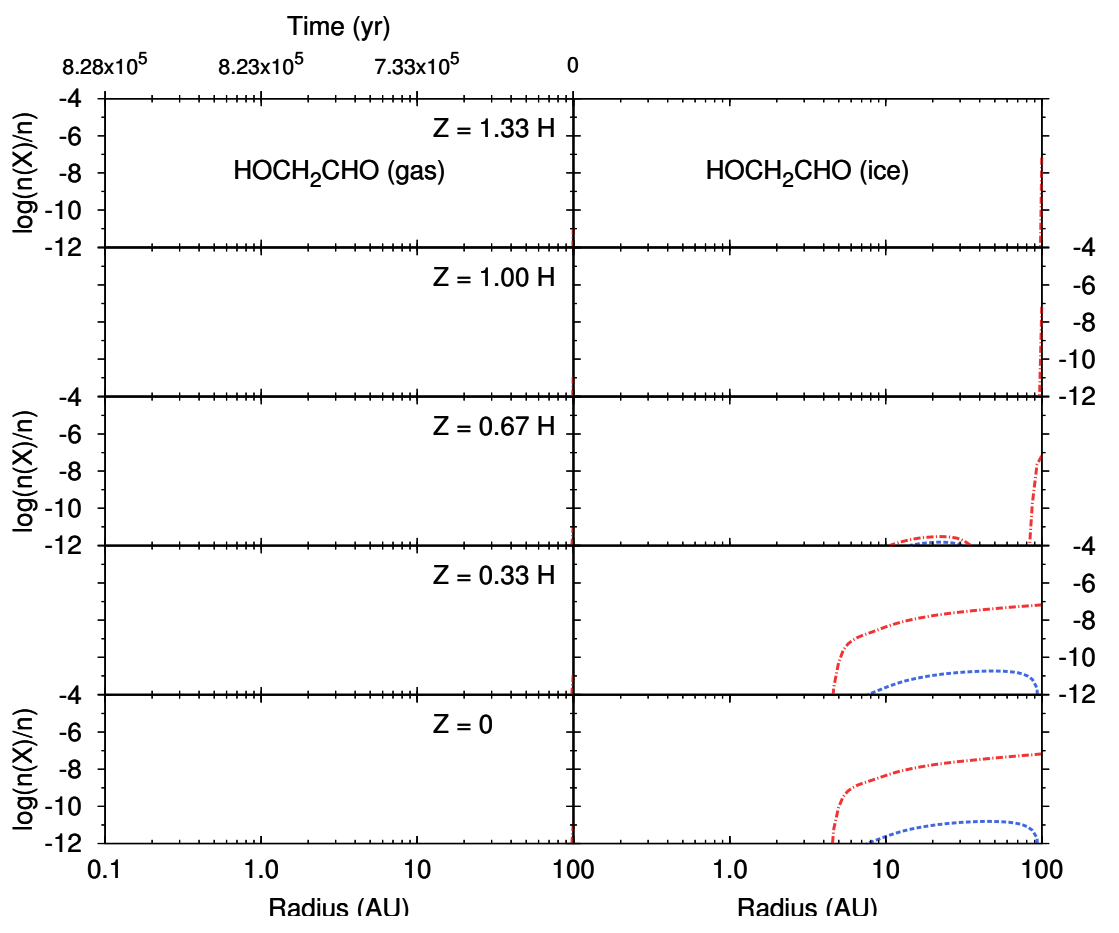

30 | Faraday Discuss., [year], [vol], $1-36$

This journal is @ The Royal Society of Chemistry [year] 
Recently, the chemical evolution along parcels of gas in a hydrodynamical simulation of a gravitationally unstable embedded disk were reported for the first time. 100 The parcels were found to follow erratic paths encountering a range of physical conditions and spikes in temperature and density due to shocks. The resulting molecular abundances trace density enhancements in spiral arms and a subsequent calculation of the molecular line emission suggested these structures may be observable with modern interferometric facilities such as ALMA.101 Again, grain-surface chemistry was not included in these calculations, and the influence on the abundance and distribution of COMs in hydrodynamic simulations of protoplanetary disks is yet to be investigated.

There are several additional physical processes thought to be important in disks which can influence the physical structure and resulting chemistry. ${ }^{[102]}$ The effects of turbulent mixing on the composition of protoplanetary disks has been

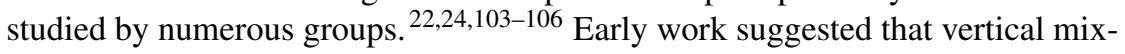
ing does not change the chemically stratified nature of the disk; however, mixing can significantly increase the depth of the molecular layer and thus the vertical column densities. ${ }^{104}$ Of these works, only one has included the formation of complex organic molecules via thermal grain-surface chemistry. ${ }^{106}$ It was found that the abundances of COMs are very sensitive to the treatment of turbulent mixing, a similar conclusion to that presented here for the case of advection. A chemical model including both processes would be ideal; however, such models remain computationally challenging.

The dust evolution in the disk can have a profound effect on the disk physical structure and resulting chemistry. Grain growth (coagulation) skews the dustgrain size distribution towards larger grains whereas settling (sedimentation) depletes the surface layers of large grains and increases the relative abundance of large grains in the disk midplane. ${ }^{107 / 108}$ Several groups have investigated the influence of dust-grain evolution on disk chemistry. $109-113$ The inclusion of grain growth tended to push the molecular layer deeper into the disk with increasing grain size; however, the vertical column densities of molecules remained unaffected. ${ }^{110}$ Grain settling was found to lead to a smaller freeze out zone and a larger molecular layer due to the increased penetration of UV radiation. ${ }^{1111} \mathrm{Re}-$ cent models have coupled both dust evolution processes in a disk model with full gas-grain chemistry. $112[113$ In these models the chemically stratified nature of the disk is retained, albeit with a molecular layer which resides deeper into the disk. Both works also found that the gas-phase column densities are enhanced (at the expense of the grain-surface column densities) relative to the results using pristine interstellar dust. Grain coagulation may affect the formation rate of COMs due to the reduced surface area available for freeze out of the necessary precursor species. On the other hand, grain settling increases the dust-to-gas mass ratio in the disk midplane relative to that higher in the disk atmosphere, somewhat counteracting the reduction in total surface area. In addition, the deeper penetration of UV radiation may increase the efficiency of both thermal and non-thermal desorption of COMs formed on the grain into the gas phase. The combined effect of accretion flow and dust evolution on the abundance and distribution of COMs in protoplanetary disks should be explored in the future.

This journal is ( The Royal Society of Chemistry [year]

Faraday Discuss., [year], [vol], 1-36 | 31 


\section{Conclusions}

The results presented here suggest that COMs which have efficiently formed on grain surfaces under cold, i.e., prestellar, conditions survive the transport along the accretion flow in the midplane of isolated disks; hence, the composition of icy planetesimals in isolated disks may be representative of the pristine interstellar ice entering the outer shielded regions of the disk, provided these species reach appreciable abundances under prestellar conditions. This conclusion is similar to that reached for simple ice species, e.g., $\mathrm{H}_{2} \mathrm{O}$, in investigations on the influence of collapse, infall, and subsequent disk formation in low-mass protostars 1144 Currently, a similar investigation is underway for more complex ice species. ${ }^{[115]}$ The successful identification of gas-phase COMs such as $\mathrm{CH}_{3} \mathrm{OH}$, $\mathrm{CH}_{3} \mathrm{CHO}, \mathrm{HCOOCH}_{3}$, and $\mathrm{CH}_{3} \mathrm{OCH}_{3}$ in dark clouds and prestellar cores also suggest that grain-surface chemistry can operate effectively in dense, cold environments. ${ }^{96]} \sqrt{98}$ However, it should be noted that, of the species considered in this work, only methanol ice and formic acid ice have been detected in prestellar environments. $43|95| 116-119$

If simple ices only are injected into the outer disk, the physical conditions facilitate the efficient production of COMs such as those considered here: $\mathrm{CH}_{3} \mathrm{CHO}$, $\mathrm{HCOOCH}_{3}, \mathrm{CH}_{3} \mathrm{OCH}_{3}$, and $\mathrm{HOCH}_{2} \mathrm{CHO}$. Hence, there is an increase in molecular complexity from cloud to disk, producing abundances of complex molecules similar to those observed in cometary comae. $\frac{120}{120}$ This conclusion is similar to that reached in the static disk model. $\frac{19}{19}$ The chemical timescales are sufficiently short in the outer disk that the results from the static model and accretion model (presented here) are similar but not identical. The fractional abundances of grainsurface COMs along the disk midplane in the static model vary with radius: the long lifetime of the disk allows the chemistry to settle to the local physical conditions. Hence, the assumed initial abundance of COMs are not preserved in the static model as is seen in the accretion flow model presented here. This work has shown that the grain-surface chemistry is sensitive to both the initial abundances adopted in the model and the accretion flow.

Once the snowline of each species is breached, the COMs can thermally desorb with the gas-phase abundance reflecting that injected from the ice mantle as also found in previous work investigating the transport of methanol ice in a disk. ${ }^{23}$ This is in stark contrast to that found in the static model in which the extreme densities and temperatures encountered in the inner midplane are able to destroy gas-phase COMs over the lifetime of the disk $\sim 10^{6}$ years. In this model, the gas spends only $\approx 5000$ years within $1 \mathrm{AU}$ of the star. In order to properly treat the transport and chemistry of complex molecules within the planet-forming region of isolated protoplanetary disks, the influence of the accretion flow must be taken into account. In the disk molecular layer, COMs photodesorb into the gas phase, reaching peak fractional abundances similar to those found in the static model. 19

Conversely, the radiation field in the irradiated disk is too strong for photodesorbed molecules to survive in the gas phase. It is also found that the assumed initial abundances are more important for this model due to the much higher temperatures in the outer disk. If simple ices are injected, thermal grain-surface chemistry is unable to form complex molecules because the necessary precursor

32 | Faraday Discuss., [year], [vol], 1-36

This journal is @ The Royal Society of Chemistry [year] 
radicals, which tend to be particularly volatile, can desorb efficiently. If more complex ices are injected into the outer disk, the grain-surface COMs undergo thermal processing such that the gas phase abundances in the inner region do not reflect those injected at the outer edge of the disk. The origin of gas-phase COMs in the inner region of the irradiated disk is gas-phase formation induced by the thermal desorption of strongly bound molecules,such as $\mathrm{CH}_{3} \mathrm{OH}$, which do not rely on radical-radical formation. This could mean that icy planetesimals which form in the midplane of irradiated disks are likely to be more thermally processed and also composed of more simple ices than those in isolated disks. This is interesting given that the Sun has been postulated to have formed in a stellar cluster which has long since dispersed. ${ }^{5}$ If the comets in the Solar System were originally composed of more simple ices, their current composition could reflect post-formation processing of the cometary surface.121

The work presented here does not address one of the outstanding issues in grain-surface chemistry, that is, the efficient formation at $10 \mathrm{~K}$ of those complex molecules which are thought to form via radical-radical association reactions. Under the current paradigm, molecular radicals do not have sufficient mobility to diffuse within or on the ice mantle. There are several processes which have been postulated to help build chemical complexity at $10 \mathrm{~K}$ including the radiationprocessing of ices via UV photons and cosmic rays, $\frac{50 \mid 122}{}$ and a high efficiency of reactive desorption. $123 \mid 124$ These issues will be explored, in the context of protoplanetary disk chemistry, in future work.

\section{Acknowledgements}

C.W. acknowledges support from the European Union A-ERC grant 291141 CHEMPLAN and financial support (program number 639.041.335) from the Netherlands Organisation for Scientific Research (NWO). EH wishes to acknowledge the support of the National Science Foundation for his astrochemistry program, and his program in chemical kinetics through the Center for the Chemistry of the Universe. He also acknowledges support from the NASA Exobiology and Evolutionary Biology program through a subcontract from Rensselaer Polytechnic Institute. H. N. acknowledges the Grant-in-Aid for Scientific Research 23103005 and 25400229. She also acknowledges support from the Astrobiology Project of the CNSI, NINS (Grant Number AB251002, AB251012). Astrophysics at QUB is supported by a grant from the STFC. S.L.W.W. acknowledges support from startup funds provided by Emory University.

\section{References}

1 F. H. Shu, F. C. Adams and S. Lizano, Annu. Rev. Astron. Astrophys., 1987, 25, 23.

2 J. P. Williams and L. A. Cieza, Annu. Rev. Astron. Astrophys., 2011, 49, 67.

3 C. J. Lada and E. A. Lada, Annu. Rev. Astron. Astrophys., 2003, 41, 57.

4 J. H. Kastner, B. Zuckerman, D. A. Weintraub and T. Forveille, Science, 1997, $277,67$.

5 F. C. Adams, Annu. Rev. Astron. Astrophys., 2010, 48, 47.

6 C. R. O'dell, Z. Wen and X. Hu, Astrophys. J., 1993, 410, 696.

7 J. Bally, R. S. Sutherland, D. Devine and D. Johnstone, Astron. J., 1998, 116, 293.

8 J. Blum and G. Wurm, Annu. Rev. Astron. Astrophys., 2008, 46, 21.

9 F. G. Bridges, K. D. Supulver, D. N. C. Lin, R. Knight and M. Zafra, Icarus, 1996, 123, 422.

This journal is ( ) The Royal Society of Chemistry [year]

Faraday Discuss., [year], [vol], 1- 36 | 33 
10 K. D. Supulver, F. G. Bridges, S. Tiscareno, J. Lievore and D. N. C. Lin, Icarus, 1997, 129, 539.

11 B. Gundlach, S. Kilias, E. Beitz and J. Blum, Icarus, 2011, 214, 717.

12 E. Herbst and E. F. van Dishoeck, Annu. Rev. Astron. Astrophys., 2009, 47, 427.

13 M. P. Bernstein, J. P. Dworkin, S. A. Sandford, G. W. Cooper and L. J. Allamandola, Nature, 2002, 416, 401.

14 G. M. Muñoz Caro, U. J. Meierhenrich, W. A. Schutte, B. Barbier, A. Arcones Segovia, H. Rosenbauer, W. H.-P. Thiemann, A. Brack and J. M. Greenberg, Nature, 2002, 416, 403.

15 D. E. Woon, Astrophys. J., 2002, 571, L177.

16 S. J. Kenyon and L. Hartmann, Astrophys. J., 1987, 323, 714.

17 E. I. Chiang and P. Goldreich, Astrophys. J., 1997, 490, 368.

18 P. D'Alessio, J. Canto, N. Calvet and S. Lizano, Astrophys. J., 1998, 500, 411.

19 C. Walsh, T. J. Millar, H. Nomura, E. Herbst, S. Widicus Weaver, Y. Aikawa, J. C. Laas and A. I. Vasyunin, Astron. Astrophys., 2014, 563, A33.

20 C. Walsh, T. J. Millar and H. Nomura, Astrophys. J., 2013, 766, L23.

21 Y. Aikawa, T. Umebayashi, T. Nakano and S. M. Miyama, Astrophys. J., 1999, 519, 705.

22 M. Ilgner, T. Henning, A. J. Markwick and T. J. Millar, Astron. Astrophys., 2004, 415, 643.

23 H. Nomura, Y. Aikawa, Y. Nakagawa and T. J. Millar, Astron. Astrophys., 2009, 495, 183.

24 D. Heinzeller, H. Nomura, C. Walsh and T. J. Millar, Astrophys. J., 2011, 731, 115.

25 E. A. Bergin, Y. Aikawa, G. A. Blake and E. F. van Dishoeck, Protostars and Planets V, 2007, 751.

26 K. I. Öberg, R. Murray-Clay and E. A. Bergin, Astrophys. J., 2011, 743, L16.

27 P. Caselli and C. Ceccarelli, Astron. Astrophysics. Rev., 2012, 20, 56.

28 A. Dutrey, S. Guilloteau and M. Guelin, Astron. Astrophys., 1997, 317, L55.

29 G.-J. van Zadelhoff, E. F. van Dishoeck, W.-F. Thi and G. A. Blake, Astron. Astrophys., 2001, $377,566$.

30 C. Qi, J. E. Kessler, D. W. Koerner, A. I. Sargent and G. A. Blake, Astrophys. J., 2003, 597, 986.

31 E. F. van Dishoeck, W.-F. Thi and G.-J. van Zadelhoff, Astron. Astrophys., 2003, 400, L1.

32 W.-F. Thi, G.-J. van Zadelhoff and E. F. van Dishoeck, Astron. Astrophys., 2004, 425, 955.

33 S. Guilloteau, V. Piétu, A. Dutrey and M. Guélin, Astron. Astrophys., 2006, 448, L5.

34 C. Qi, D. J. Wilner, Y. Aikawa, G. A. Blake and M. R. Hogerheijde, Astrophys. J., 2008, 681, 1396.

35 K. I. Öberg, C. Qi, J. K. J. Fogel, E. A. Bergin, S. M. Andrews, C. Espaillat, T. A. van Kempen, D. J. Wilner and I. Pascucci, Astrophys. J., 2010, 720, 480.

36 Y. Aikawa, M. Momose, W.-F. Thi, G.-J. van Zadelhoff, C. Qi, G. A. Blake and E. F. van Dishoeck, Pub. Astron. Soc. Japan, 2003, 55, 11.

37 E. Chapillon, A. Dutrey, S. Guilloteau, V. Piétu, V. Wakelam, F. Hersant, F. Gueth, T. Henning, R. Launhardt, K. Schreyer and D. Semenov, Astrophys. J., 2012, 756, 58.

38 C. Qi, K. I. Öberg, D. J. Wilner and K. A. Rosenfeld, Astrophys. J., 2013, 765, L14.

39 M. Allen and G. W. Robinson, Astrophys. J., 1977, 212, 396.

40 D. E. Woon, Astrophys. J., 2002, 569, 541.

41 N. Watanabe and A. Kouchi, Astrophys. J., 2002, 571, L173.

42 G. W. Fuchs, H. M. Cuppen, S. Ioppolo, C. Romanzin, S. E. Bisschop, S. Andersson, E. F. van Dishoeck and H. Linnartz, Astron. Astrophys., 2009, 505, 629.

43 E. L. Gibb, D. C. B. Whittet, A. C. A. Boogert and A. G. G. M. Tielens, Astrophys. J., Suppl. Ser., 2004, 151, 35.

44 R. T. Garrod and E. Herbst, Astron. Astrophys., 2006, 457, 927.

45 R. T. Garrod, S. L. W. Weaver and E. Herbst, Astrophys. J., 2008, 682, 283.

46 J. C. Laas, R. T. Garrod, E. Herbst and S. L. Widicus Weaver, Astrophys. J., 2011, 728, 71.

47 S. S. Prasad and S. P. Tarafdar, Astrophys. J., 1983, 267, 603.

48 L. J. Allamandola, S. A. Sandford and G. J. Valero, Icarus, 1988, 76, 225.

49 P. A. Gerakines, W. A. Schutte and P. Ehrenfreund, Astron. Astrophys., 1996, 312, 289.

50 K. I. Öberg, R. T. Garrod, E. F. van Dishoeck and H. Linnartz, Astron. Astrophys., 2009, 504, 891.

51 M. S. Westley, R. A. Baragiola, R. E. Johnson and G. A. Baratta, Planet. Space Sci., 1995, 43, 1311. 
52 K. Willacy and W. D. Langer, Astrophys. J., 2000, 544, 903.

53 C. Walsh, T. J. Millar and H. Nomura, Astrophys. J., 2010, 722, 1607.

54 M. Bertin, E. C. Fayolle, C. Romanzin, K. I. Öberg, X. Michaut, A. Moudens, L. Philippe, P. Jeseck, H. Linnartz and J.-H. Fillion, Phys. Chem. Chem. Phys., 2012, 14, 9929.

55 K. I. Öberg, H. Linnartz, R. Visser and E. F. van Dishoeck, Astrophys. J., 2009, 693, 1209.

56 C. Arasa, S. Andersson, H. M. Cuppen, E. F. van Dishoeck and G.-J. Kroes, J. Chem. Phys., 2010, 132, 184510.

57 M. R. Hogerheijde, E. A. Bergin, C. Brinch, L. I. Cleeves, J. K. J. Fogel, G. A. Blake, C. Dominik, D. C. Lis, G. Melnick, D. Neufeld, O. Panić, J. C. Pearson, L. Kristensen, U. A. Yıldız and E. F. van Dishoeck, Science, 2011, 334, 338.

58 M. J. Mumma, P. R. Weissman and S. A. Stern, Protostars and Planets III, 1993, p. 1177.

59 H. Nomura and T. J. Millar, Astron. Astrophys., 2005, 438, 923.

60 H. Nomura, Y. Aikawa, M. Tsujimoto, Y. Nakagawa and T. J. Millar, Astrophys. J., 2007, 661, 334.

61 S. J. Kenyon and L. Hartmann, Astrophys. J., Suppl. Ser., 1995, 101, 117.

62 J. E. Pringle, Annu. Rev. Astron. Astrophys., 1981, 19, 137.

63 H. Nomura, Astrophys. J., 2002, 567, 587.

64 D. N. C. Lin and J. Papaloizou, Mon. Not. R. Astron. Soc., 1980, 191, 37.

65 G. H. Herbig and R. W. Goodrich, Astrophys. J., 1986, 309, 294.

66 E. D. Feigelson and T. Montmerle, Annu. Rev. Astron. Astrophys., 1999, 37, 363.

67 J. C. Weingartner and B. T. Draine, Astrophys. J., 2001, 548, 296.

68 P. R. Maloney, D. J. Hollenbach and A. G. G. M. Tielens, Astrophys. J., 1996, 466, 561.

69 E. F. van Dishoeck, Astrochemistry, 1987, p. 51.

70 E. F. van Dishoeck, B. Jonkheid and M. C. van Hemert, Faraday Discussions, 2006, 133, 231.

71 A. E. Glassgold, P. J. Huggins and W. D. Langer, Astrophys. J., 1985, 290, 615.

72 H.-H. Lee, E. Herbst, G. Pineau des Forets, E. Roueff and J. Le Bourlot, Astron. Astrophys., 1996, 311, 690.

73 R. Visser, E. F. van Dishoeck and J. H. Black, Astron. Astrophys., 2009, 503, 323.

74 X. Li, A. N. Heays, R. Visser, W. Ubachs, B. R. Lewis, S. T. Gibson and E. F. van Dishoeck, Astron. Astrophys., 2013, 555, A14.

75 E. Matar, H. Bergeron, F. Dulieu, H. Chaabouni, M. Accolla and J. L. Lemaire, J. Chem. Phys., 2010, 133, 104507.

76 X. Sha, B. Jackson, D. Lemoine and B. Lepetit, J. Chem. Phys., 2005, 122, 014709.

77 H. M. Cuppen, L. E. Kristensen and E. Gavardi, Mon. Not. R. Astron. Soc., 2010, 406, L11.

78 T. I. Hasegawa, E. Herbst and C. M. Leung, Astrophys. J., Suppl. Ser., 1992, 82, 167.

79 T. I. Hasegawa and E. Herbst, Mon. Not. R. Astron. Soc., 1993, 261, 83.

80 K. I. Öberg, G. W. Fuchs, Z. Awad, H. J. Fraser, S. Schlemmer, E. F. van Dishoeck and H. Linnartz, Astrophys. J., 2007, 662, L23.

81 K. I. Öberg, E. F. van Dishoeck and H. Linnartz, Astron. Astrophys., 2009, 496, 281.

82 T. Stantcheva, P. Caselli and E. Herbst, Astron. Astrophys., 2001, 375, 673.

83 R. T. Garrod, A. I. Vasyunin, D. A. Semenov, D. S. Wiebe and T. Henning, Astrophys. J., 2009, 700, L43.

84 A. I. Vasyunin, D. A. Semenov, D. S. Wiebe and T. Henning, Astrophys. J., 2009, $691,1459$.

85 N. Watanabe, Y. Kimura, A. Kouchi, T. Chigai, T. Hama and V. Pirronello, Astrophys. J., 2010, 714, L233.

86 R. T. Garrod, V. Wakelam and E. Herbst, Astron. Astrophys., 2007, 467, 1103.

87 C. Gry, F. Boulanger, C. Nehmé, G. Pineau des Forêts, E. Habart and E. Falgarone, Astron. Astrophys., 2002, 391, 675.

88 E. Habart, F. Boulanger, L. Verstraete, G. Pineau des Forêts, E. Falgarone and A. Abergel, Astron. Astrophys., 2003, 397, 623.

89 S. Cazaux and A. G. G. M. Tielens, Astrophys. J., 2004, 604, 222.

90 W. Iqbal, K. Acharyya and E. Herbst, Astrophys. J., 2012, 751, 58.

91 J. Le Bourlot, F. Le Petit, C. Pinto, E. Roueff and F. Roy, Astron. Astrophys., 2012, 541, A76.

92 C. Walsh, H. Nomura, T. J. Millar and Y. Aikawa, Astrophys. J., 2012, 747, 114.

93 S. Ioppolo, H. M. Cuppen, E. F. van Dishoeck and H. Linnartz, Mon. Not. R. Astron. Soc., 2011, 410, 1089. 
94 W.-C. Chen and R. A. Marcus, J. Chem. Phys., 2005, 123, 094307.

95 A. C. A. Boogert, K. M. Pontoppidan, C. Knez, F. Lahuis, J. Kessler-Silacci, E. F. van Dishoeck, G. A. Blake, J.-C. Augereau, S. E. Bisschop, S. Bottinelli, T. Y. Brooke, J. Brown, A. Crapsi, N. J. Evans, II, H. J. Fraser, V. Geers, T. L. Huard, J. K. Jørgensen, K. I. Öberg, L. E. Allen, P. M. Harvey, D. W. Koerner, L. G. Mundy, D. L. Padgett, A. I. Sargent and K. R. Stapelfeldt, Astrophys. J., 2008, 678, 985.

96 K. I. Öberg, S. Bottinelli, J. K. Jørgensen and E. F. van Dishoeck, Astrophys. J., 2010, 716, 825.

97 A. Bacmann, V. Taquet, A. Faure, C. Kahane and C. Ceccarelli, Astron. Astrophys., 2012, 541, L12.

98 J. Cernicharo, N. Marcelino, E. Roueff, M. Gerin, A. Jiménez-Escobar and G. M. Muñoz Caro, Astrophys. J., 2012, 759, L43.

99 J. K. Jørgensen, C. Favre, S. E. Bisschop, T. L. Bourke, E. F. van Dishoeck and M. Schmalzl, Astrophys. J., 2012, 757, L4.

100 J. D. Ilee, A. C. Boley, P. Caselli, R. H. Durisen, T. W. Hartquist and J. M. C. Rawlings, Mon. Not. R. Astron. Soc., 2011, 417, 2950.

101 T. A. Douglas, P. Caselli, J. D. Ilee, A. C. Boley, T. W. Hartquist, R. H. Durisen and J. M. C. Rawlings, Mon. Not. R. Astron. Soc., 2013, 433, 2064.

102 T. Henning and D. Semenov, Chemical Reviews, 2013, 113, 9016.

103 D. Semenov, D. Wiebe and T. Henning, Astrophys. J., 2006, 647, L57.

104 K. Willacy, W. Langer, M. Allen and G. Bryden, Astrophys. J., 2006, 644, 1202.

105 Y. Aikawa, Astrophys. J., 2007, 656, L93.

106 D. Semenov and D. Wiebe, Astrophys. J., Suppl. Ser., 2011, 196, 25.

107 C. Dominik and A. G. G. M. Tielens, Astrophys. J., 1997, 480, 647.

108 C. P. Dullemond and C. Dominik, Astron. Astrophys., 2004, 421, 1075.

109 B. Jonkheid, F. G. A. Faas, G.-J. van Zadelhoff and E. F. van Dishoeck, Astron. Astrophys., 2004, 428, 511.

110 Y. Aikawa and H. Nomura, Astrophys. J., 2006, 642, 1152.

111 J. K. J. Fogel, T. J. Bethell, E. A. Bergin, N. Calvet and D. Semenov, Astrophys. J., 2011, 726, 29.

112 A. I. Vasyunin, D. S. Wiebe, T. Birnstiel, S. Zhukovska, T. Henning and C. P. Dullemond, Astrophys. J., 2011, 727, 76.

113 V. Akimkin, S. Zhukovska, D. Wiebe, D. Semenov, Y. Pavlyuchenkov, A. Vasyunin, T. Birnstiel and T. Henning, Astrophys. J., 2013, 766, 8.

114 R. Visser, E. F. van Dishoeck, S. D. Doty and C. P. Dullemond, Astron. Astrophys., 2009, 495, 881.

115 M. Drozdovskaya, C. Walsh, R. Visser, D. Harsono and E. F. van Dishoeck, Mon. Not. R. Astron. Soc., 2014, submitted.

116 W. A. Schutte, A. C. A. Boogert, A. G. G. M. Tielens, D. C. B. Whittet, P. A. Gerakines, J. E. Chiar, P. Ehrenfreund, J. M. Greenberg, E. F. van Dishoeck and T. de Graauw, Astron. Astrophys., 1999, 343, 966.

117 E. L. Gibb, D. C. B. Whittet, W. A. Schutte, A. C. A. Boogert, J. E. Chiar, P. Ehrenfreund, P. A. Gerakines, J. V. Keane, A. G. G. M. Tielens, E. F. van Dishoeck and O. Kerkhof, Astrophys. J., 2000, 536, 347.

118 K. M. Pontoppidan, E. Dartois, E. F. van Dishoeck, W.-F. Thi and L. d'Hendecourt, Astron. Astrophys., 2003, 404, L17.

119 A. C. A. Boogert, T. L. Huard, A. M. Cook, J. E. Chiar, C. Knez, L. Decin, G. A. Blake, A. G. G. M. Tielens and E. F. van Dishoeck, Astrophys. J., 2011, 729, 92.

120 D. Bockelée-Morvan, J. Crovisier, M. J. Mumma and H. A. Weaver, in The composition of cometary volatiles, ed. M. C. Festou, H. U. Keller and H. A. Weaver, University of Arizona Press, Tucson, 2004, p. 391.

121 M. J. Mumma and S. B. Charnley, Annu. Rev. Astron. Astrophys., 2011, 49, 471.

122 C. J. Bennett and R. I. Kaiser, Astrophys. J., 2007, 661, 899.

123 A. I. Vasyunin and E. Herbst, Astrophys. J., 2013, 769, 34.

124 F. Dulieu, E. Congiu, J. Noble, S. Baouche, H. Chaabouni, A. Moudens, M. Minissale and S. Cazaux, Scientific Reports, 2013, 3, 1338.

36 | Faraday Discuss., [year], [vol], 1-36

This journal is @ The Royal Society of Chemistry [year] 